\title{
Country centrality in the international multiplex network
}

\author{
Giovanni Bonaccorsi $1^{*}$ (D) Massimo Riccaboni ${ }^{1}$, Giorgio Fagiolo ${ }^{2}$ and Gianluca Santoni ${ }^{3}$
}

\author{
*Correspondence: \\ giovanni.bonaccorsi@imtlucca.it \\ 'IMT School of Advanced Studies, \\ Lucca, 55100, Lucca, Italy \\ Full list of author information is \\ available at the end of the article
}

\begin{abstract}
In this work we introduce and analyze a new and comprehensive multilayer dataset covering a wide spectrum of international relationships between coutries. We select two cross sections of the dataset corresponding to years 2003 and 2010 with 19 layers and 112 nodes to study the structure and evolution of the network. Country centrality is measured by the multiplex PageRank (MultiRank) and the multiplex hub and authority scores (MultiHub and MultiAuth). We find that the MultiHub measure has the highest correlation to GDP per capita, with respect to the other multilayer measures and to their single layer analogues. Finally we analyze the differences in the ranking between GDP per capita and the multilayer centrality measures to evaluate them as measures of development.
\end{abstract}

Keywords: Multiplex, Centrality, Development, MultiRank, MD-HITS, International exchanges

\section{Introduction}

Over the last few years we have witnessed an increase in the use of network models in economics, finance and business studies: details coming from the topology of specific networks, either on micro level of social interactions among individuals and collaborations between firms, or at a macro level of international economics, help scholars identify new phenomena and clarify how they diffuse and distribute (Easley and Kleinberg 2010).

Usually, though, one network is examined at a time (i.e. trade) or the relationship between two networks is investigated (i.e. migration and trade). Network features are frequently used as explanatory or descriptive variables in gravity models of international activities. For instance, we have several studies on the effect of the centrality of countries in the trade and migration networks on the magnitude of countries' exports (Fagiolo and Mastrorillo 2014; Sgrignoli et al. 2015; Metulini et al. 2018). Occasionally, other networks have been considered in the literature, such as the network of foreign direct investments, human mobility, information, knowledge and financial flows.

On the one hand, country centrality in networks has been used in the study of international relations as a proxy of power (Hafner-Burton et al. 2009). On the other hand, new developments in multilayer network analysis suggest that international studies may greatly benefit from jointly analyzing multiple international relations in a unified framework (De Domenico et al. 2015b).

In this paper we study the international network of countries by looking at several dimensions of development from a multilayer network perspective. We assemble a mul-

(c) The Author(s). 2019 Open Access This article is distributed under the terms of the Creative Commons Attribution 4.0 International License (http://creativecommons.org/licenses/by/4.0/), which permits unrestricted use, distribution, and reproduction in any medium, provided you give appropriate credit to the original author(s) and the source, provide a link to the Creative Commons license, and indicate if changes were made. 
tiplex network of 112 countries as nodes and their connections across 19 heterogeneous layers, observed at two moments in time before and after the Great Recession (2003 and 2010). We compute the centrality of countries in the multilayer network by using the multilayer generalization of the PageRank algorithm (Iacovacci and Bianconi 2016) MultiRank from now on - and of the hubs and authority score (Arrigo and Tudisco 2018) - MD-HITS from now on.

The ranking we obtain based on MultiRank centrality is consistent with the common north-south divide, that is to say the division of countries among developed and underdeveloped, and depicts a positive correlation with relevant economic variables such as GDP per capita purchasing power parity (PPP) (pcGDP from now on). This configuration of MultiRank gives more importance to those layers which are commonly considered as relevant for the performance of countries, predominantly trade and financial networks. Moreover it is very similar to the ranking selected by the MD-HITS algorithm, with the latter actually having an even better fit with relevant macroeconomic variables.

Our work sheds new light on the relationship between position and centrality of countries in the international multiplex network and their development by analyzing, for the first time, a vast set of international relations: trade, finance, transportation, human mobility and migrations, information and knowledge flows, international alliances. Increasing data availability coupled with the recent advancements in the analysis of multiple networks open up new possibilities for the analysis of complex global phenomena and new dimensions can be added to our framework to cover an even broader set of international linkages.

The rest of the paper is structured as follows: "Related literature" section describes how our work fits in the extant literature, "Data" section contains a detailed description of the data we have collected, "Multiplex centrality" section explains the methodology we apply and finally "Results" section illustrates our results. Finally the last section discusses our contribution to the literature and future work.

\section{Related literature}

Following previous applications of network theory in macroeconomics, we define a single layer economic network $G(N, E)$ as a set of nodes $N$ representing countries, coupled with a set of edges $E$ describing the relations between them. In so doing we follow a long tradition of studies in trade (Chaney 2014), migration (Fagiolo and Mastrorillo 2014), international aid (Kali et al. 2017), banking (Minoiu and Reyes 2013; Battiston et al. 2012; Bardoscia et al. 2015), mergers and acquisitions (Campi et al. 2016). Usually, the edges between nodes are weighted and directed, representing the amount of monetary exchanges from a source country to a target country.

However, there are some international networks in which directed edges represent relationships other than monetary exchanges, such as the networks of migration and human mobility (Sgrignoli et al. 2015; Fagiolo and Santoni 2016; Fagiolo and Santoni 2015; Fagiolo and Mastrorillo 2014; Riccaboni et al. 2013).

Sometimes edges may also be undirected (symmetric) and unweighted. A stream of literature has investigated international infrastructures including, among others, the international airport transportation network (Colizza et al. 2006), the trade shipping network (Kaluza et al. 2010) and the overseas telephone and fiber cables linkage (Rossello 2015). Intangible networks of knowledge flows have also been studied by looking at 
patent citations (Hall et al. 2001) and international collaboration among scholars (Pan et al. 2012). Finally, international relationships are shaped by the networks of diplomatic relationships, such as alliances, trade agreements and intergovernmental organization memberships, as reported in the "Correlates of war" project (Bayer 2006; Pevehouse et al. 2003; Gibler 2008; de Sousa and Lochard 2011).

Bulding upon previous studies, which have analyzed the different dimensions of international connectivity, and thanks to multiple available data sources, in this paper we reconstruct the first comprehensive multilayer network among countries: instead of analyzing each of the layers in isolation, focusing on standard network statistics and comparing them, we analyze the structure of international networks considered as a whole. Following Kivela et al. (2014) we define our multilayer network as a multiplex, i.e. a network where no link connects directly the layers but all the layers share the same set of nodes, e.g. countries. Therefore, a multiplex is a colored network where edges of different colors represent different types of international relationships (i.e. trade, migration, investments, knowledge flows etc) between countries.

In recent years multilayer networks have become the subject of several scholarly works aimed at generalizing network statistics and algorithms from single to multiple layer analysis (Lee et al. 2012; De Domenico et al. 2015a; Kivela et al. 2014; Battiston et al. 2014; Boccaletti et al. 2014; Bródka et al. 2018; De Bacco et al. 2017; Aleta and Moreno 2019).

Among the numerous methods which have been proposed to measure the centrality of nodes in a multilayer network we have selected two measures which can be computed for weighted and directed multilayer networks and do not require any pre-defined ranking of the importance of network layers (i.e. different types of international relationships), but provide it as an output.

The first is the generalization to multiplex network of the PageRank centrality of nodes (Brin and Page 1998). According to it, nodes are ranked higher if they receive links from other important nodes: hence it focuses on the attractiveness of nodes only. To apply it to our multilayer network we closely followed a set of recently published papers (Rahmede et al. 2018; Halu et al. 2013; Iacovacci et al. 2016; Iacovacci and Bianconi 2016) ${ }^{1}$ which have developed a way of defining the attractiveness of nodes by taking into account also the relevance of each layer in the whole multiplex. In the multiplex version of the Pagerank nodes are rewarded for their capacity of receiving links from other central nodes in more relevant layers.

Our second choice is the hubs and authority algorithm, also known as HITS, HyperlinkInduced Topic Search, (Kleinberg 1999). Referring to the problem of getting access to information, it defines as authorities those nodes which hold essential information to the user whereas hubs are those nodes which redirect the user to the authorities ${ }^{2}$. In our case some countries act as brokers among important partners whereas other countries acquire importance for being connected to many hubs. To apply this algorithm to our data we follow the work by Arrigo and Tudisco (2018) where not only nodes

\footnotetext{
${ }^{1}$ Specifically we apply the version of the algorithm where both layer relevance and node PageRank are calculated at each iteration and for multiplexes of many layers.

${ }^{2}$ The classification reflects how information is searched and found: it is difficult to find the right answer at first try, hence there are nodes which collect information and redirect the user to the right destination gaining in centrality for this reason. Hence to have high authority score a node must have links from nodes with high hub score, whereas higher hub score is awarded to nodes linking to high authorities nodes.
} 
are ranked but also layers receive a score, ranking their capacity of linking important layers or being linked by hubs layers ${ }^{3}$.

One challenge we face is to analyze a multiplex of heterogeneous layers to characterize the relevance of countries over a set of different features (flows of goods, services, investments, knowledge, human mobility and so on). ${ }^{4}$ So far, only multiplexes of homogeneous layers have been considered, where each layer represents a different typology of the same relation (different trade commodities in the trade network, different airlines in the airport network and so on). To deal with this issue we have filtered our data both by node, i.e. by deciding which nodes were constantly measured over time, and by edge, i.e. by selecting only relevant edges and discarding less probable ones.

The position and role of countries in the multiple network of international relations is then compared to pcGDP as a measure to summarize the relative economic performance of countries. Our work also contributes to the ongoing debate on the appropriateness of GDP per capita as a measure of the well being of populations (Sen 1985; Dasgupta and Mäler 2000; Stevenson and Wolfers 2013; Lange and Wodon 2018). Alternative measures could include relevant information on the role a country plays in the multiple networks of global relations.

\section{Data}

As acknowledged in the previous section, this work takes advantage of previous applied network studies mapping international relations among countries. We have collected and integrated multiple data sources in a unique dataset spanning several years and we have selected among the different layers the ones we thought were the most significant to describe the role of a country in the global system. Figure 12 in the Appendix provides a full overview of all the data we collected for years 1990-2015, also with examples of layers which have not been included in our multiplex. In this section instead we first explain the criteria we have followed to select layers, then we describe their main structural properties and finally we describe the method we have used to normalize them. All the details about the sources we have consulted for the selected layers are reported in Table 1 with references to the literature and URL links to online sources when retrievable.

\section{A selection of international networks}

Our main objective in building the international multiplex has been to identify two cross sections with a sufficient number of nodes and layers to compare the structure of the multiplex before and after the Great Recession.

From the larger dataset showed in Fig. 12 in the Appendix we have first selected a set of layers with non overlapping characteristics, avoiding duplicates and partial sources.

The selection of the two years in the dataset to be compared is based on two criteria: first network measures often evolve slowly, thus a sufficiently long period is needed to capture some changes; second depending on the choice of the years some layers might not be present in the final selection, due to lack of data. To partially mitigate this second issue we have relaxed the constraint on some significant layers including them in the final cross sections even when they are missing in a given year, but data are available for an interval around it.

\footnotetext{
${ }^{3}$ More details on this are provided in "Multiplex centrality" section.

${ }^{4}$ By doing so our work is related to social network studies of international relationships such as Smith and White (1992).
} 
Table 1 Variable summary: descriptive statistics of the raw data

\begin{tabular}{|c|c|c|c|c|c|c|c|c|c|c|}
\hline \multirow[t]{2}{*}{ layer code } & nodes & edges & \multicolumn{4}{|c|}{ weights $^{a}$} & \multirow[t]{2}{*}{$U^{b}$} & \multirow[t]{2}{*}{$\mathrm{s}^{c}$} & \multirow[t]{2}{*}{$t_{0}$} & \multirow[t]{2}{*}{$t_{1}$} \\
\hline & $t_{0} \quad t_{1}$ & $t_{0} \quad t_{1}$ & $\min$ & $\max$ & avg. & std. & & & & \\
\hline fta_wto & $211 / 211$ & $2800 / 5004$ & $1.00 \mathrm{E}+00$ & $1.00 \mathrm{E}+00$ & $1.00 \mathrm{E}+00$ & $0.00 \mathrm{E}+00$ & $\checkmark$ & $\checkmark$ & 2003 & 2010 \\
\hline expv & $211 / 211$ & $24276 / 25862$ & $1.00 \mathrm{E}+00$ & $3.49 \mathrm{E}+08$ & $4.24 E+05$ & $4.24 \mathrm{E}+06$ & & & 2003 & 2010 \\
\hline serv_exp & 207 / 207 & $4903 / 7126$ & $1.00 E+00$ & $5.72 E+10$ & $3.33 E+08$ & $1.92 \mathrm{E}+09$ & & & 2003 & 2010 \\
\hline arms & $153 / 153$ & $274 / 396$ & $1.00 \mathrm{E}+00$ & $2.33 \mathrm{E}+03$ & $6.60 \mathrm{E}+01$ & $1.98 \mathrm{E}+02$ & & & 2003 & 2010 \\
\hline invest & $209 / 208$ & 2892 / 3997 & $1.00 \mathrm{E}+00$ & 1.14E+06 & $6.19 \mathrm{E}+03$ & $3.72 E+04$ & & & 2003 & 2010 \\
\hline FDI & $210 / 210$ & $452 / 720$ & $0.00 \mathrm{E}+00$ & $1.25 E+05$ & $3.19 \mathrm{E}+03$ & $1.11 \mathrm{E}+04$ & & & 2005 & 2010 \\
\hline FDI_Greenfield & $158 / 158$ & $1767 / 2233$ & 7.00E-02 & 2.19E+04 & $3.95 E+02$ & $1.25 \mathrm{E}+03$ & & & 2003 & 2010 \\
\hline value & $177 / 177$ & $672 / 906$ & $3.00 \mathrm{E}-03$ & 3.47E+04 & $5.01 E+02$ & $1.74 \mathrm{E}+03$ & & & 2003 & 2010 \\
\hline BIS_flow_claims & $183 / 183$ & $1993 / 2720$ & 4.00E-03 & 8.97E+05 & $3.90 E+03$ & $2.81 E+04$ & & & 2003 & 2010 \\
\hline aid & $170 / 136$ & $2726 / 2887$ & $1.00 \mathrm{E}-02$ & 4.78E+03 & $1.98 \mathrm{E}+01$ & $1.15 \mathrm{E}+02$ & & & 2006 & 2010 \\
\hline migration_flow & $179 / 179$ & 11544 / 11893 & $1.00 \mathrm{E}+00$ & $2.68 \mathrm{E}+06$ & $3.47 E+03$ & $3.24 \mathrm{E}+04$ & & & 2000 & 2005 \\
\hline migration_stock & $205 / 202$ & 10787 / 10991 & $1.00 \mathrm{E}+00$ & $1.16 \mathrm{E}+07$ & $1.81 \mathrm{E}+04$ & $1.53 \mathrm{E}+05$ & & & 2005 & 2010 \\
\hline out_tour & $189 / 191$ & 9842 / 10789 & $1.00 \mathrm{E}+00$ & $7.93 \mathrm{E}+07$ & $7.38 \mathrm{E}+04$ & $8.80 \mathrm{E}+05$ & & & 2003 & 2010 \\
\hline mobility & $191 / 189$ & $6014 / 7215$ & $1.00 \mathrm{E}+00$ & $1.26 \mathrm{E}+05$ & $3.98 \mathrm{E}+02$ & $2.82 \mathrm{E}+03$ & & & 2003 & 2010 \\
\hline citation & $196 / 195$ & 24054 / 24054 & $0.00 E+00$ & $1.16 \mathrm{E}+07$ & $2.52 E+03$ & $8.01 E+04$ & & & 2003 & 2010 \\
\hline collaboration & $191 / 189$ & 8854 / 8854 & $0.00 E+00$ & $2.04 \mathrm{E}+06$ & $8.97 E+02$ & $2.46 \mathrm{E}+04$ & & & 2003 & 2010 \\
\hline pat_cit_inv & $164 / 164$ & $2668 / 2244$ & $1.00 \mathrm{E}+00$ & $1.68 \mathrm{E}+06$ & $9.90 \mathrm{E}+02$ & $2.71 E+04$ & & & 2003 & 2010 \\
\hline totlC & $187 / 187$ & $627 / 833$ & $0.00 E+00$ & $2.91 E+01$ & 7.87E-01 & $2.42 \mathrm{E}+00$ & & $\checkmark$ & 2003 & 2010 \\
\hline cow_alliances & $124 / 112$ & $2455 / 2505$ & $1.00 \mathrm{E}+00$ & $5.00 \mathrm{E}+00$ & $1.24 \mathrm{E}+00$ & $5.26 \mathrm{E}-01$ & & & 2003 & 2010 \\
\hline
\end{tabular}

By choosing years 2003 and 2010 as reference points we obtain the results shown in the last two columns of Table $1, t_{0}$ and $t_{1}$, where we can see that the two cross sections are scattered: migration stock, FDI and aid layers are not available for the 2003 reference year ${ }^{5}$. In the rest of the paper we will occasionally refer to the cross section in 2003 as first cross section and to the crosssection in 2010 as second cross section.

Our main goal in the construction of the international multiplex was to include as many countries as possible. By looking at the column nodes of Table 1 one can see that the number of reported nodes for some layers differs across time. To obtain a stable and large set of countries we proceeded in two steps. First, we have identified non missing observations: if the node is the target or the source of at least one non-zero edge in any of the years available for a given layer it belongs to the dataset. Second we have calculated the common subset of nodes for all layers. Some layers have been discarded from the analysis since they do not have enough observations. A more detailed discussion of our two steps strategy is provided in the Appendix, section Data selection.

As a result we selected a multiplex of 19 layers with the same set of 112 nodes in each of them, sampled at two time periods around 2003 and 2010. Table 1 shows the features of our final selection and in "Summary statistics" section we describe the content of each layer. By looking at the distribution of weights and at the density of each layer we can see that, even though balancing our two cross sections on the same set of nodes and layers ensures that we can make meaningful comparisons before and after the Crisis, some further steps are required to normalize the content of the dataset, across multiple 
and heterogeneous dimensions. In "Filtering" section we explain how we have filtered the edges to make layers comparable. For additional details on how we processed the data before filtering we refer to section "Data preprocessing" in the Appendix.

\section{Summary statistics}

Table 1 reports the list of selected networks in our multiplex, with the short name of each layer used in our dataset in the first column and some summary statistics for each layer of the multiplex: number of nodes and edges in each of the cross sections analyzed, symmetry and weight checks on the edges and actual years used to construct the database.

Layers can be classified in six categories according to the type of international relationship they represent:

- trade: trade agreements among countries (fta_wto), commodity and services exchanges (expv, serv_exp) and arms transfers (arms)

- investment: foreign direct investments (FDI, FDI_Greenfield), total portfolio investments (TPI) (invest), value of mergers and acquisitions (M\&A) (value), international aid (aid) and international bank loans (BIS_flow_claims)

- human mobility: movement of individuals between states as measured by permanent migration in flows and stocks (migration_flow, migration_stock) and temporary mobility, i.e. tourism (out_tour) and students abroad (mobility)

- knowledge flows through patent citations (pat_cit_inv), citations of scientific papers (citation) and paper coauthorship (collaboration)

- common infrastructures between countries as measured by capacity of internet cable routing (tot IC)

- diplomatic relationships (cow_alliances).

Table 1 shows that trade layers are constituted mostly by monetary flows, hence they are represented by directed weighted graphs where edges are flows of money from source country to target country. The network of trade agreements ( $f t a \_w t o$ ) is one exception: it is a symmetric and unweighted layer with edges equal to 1 when two country have signed a free trade agreement.

Another exception regards arms exchange (arms): it is an interesting network to political scientist, hence a particular effort has been devoted in tracing and accounting arms trade. They come from separate sources collected in the the SIPRI database.

Financial layers too have edges representing monetary flows, such as FDI, TPI and M\&A. It is worth noticing that FDI flows are reported twice, since we integrate OECD data with a separate source (FDI_Greenfield) collected in Kirkegaard (2013) focusing on specific type of firms which are difficult to measure in the official FDI statistic. Given the extent of this second source and the relevance of its content (which differs significantly from OECD) we added it as a separate source. As for BIS flows, which represent data collected from the Bank of International Settlements on loans among international banks, we selected only the claims layer, which is also automatically adjusted for changes in exchange rates. Finally, flows of international aid constitute another exception. These specific flows of capital are named Official Development Assistance (ODA) Disbursments and are measured by the OECD. While in the other layers edges represented monetary payments from source countries to target ones, here the relation represents voluntary donation from source countries to foster the development of their counterparts. Hence 
ODA flows represent a reversed measure of the development of a countries: central nodes (in terms of incoming edges) are those which are more underdeveloped. For this reason in our final multiplex the aid layer has been reversed (more details on this in the section "Data preprocessing" in the Appendix).

The third category of layers concerns the movement of people. Layers of this group can be divided in temporary migration (tourism and residencies lasting less than one year) and permanent stock of migrants. Both measures are collected by the United Nations statistical division. A third measure, flows of migrants among countries, has been produced for every five years from 1990 to 2010 by Abel and Sander (2014) through estimates based on the UN stock data ${ }^{6}$.

Knowledge flows are represented by citations of patents and papers by countries. Patent citations come from the NBER database and refer to the country of patent inventors. Paper citations instead have been collected by Pan et al. (2012) and represent an average over a five year period. These layers have a common unit of measurement: each edge between two nodes represents the number of papers/patents of the destination country cited by the source one.

The last two layers are cable route capacity and diplomatic alliances. The first is a measure of the common telecommunication infrastructure linking two countries: each edge represents how many terabyte of data can travel between two countries via cable connection. Hence by construction this layer is weighted and symmetric. The alliances layer is symmetric too but it is unweighted: each edge represents an international diplomatic alliance between two countries. This is one of the measures of diplomatic interaction from the Correlates of War project ${ }^{7}$.

\section{Filtering}

The layers of the multiplex are very different and there is a need to standardize them before proceeding in any further analysis. In fact, the distribution of weights of layers in Table 2 (more details in Fig. 13 and 14 in the Appendix) shows that they have very different mean value, range and skewness. Moreover, in many of our sources there is a bias in the reporting countries: due to their economic (and sometimes territorial) size, developed countries take part in exchanges with other countries more frequently.

To solve these issues we apply an hypergeometric filter on the data, as in Riccaboni et al. (2013). The reasoning goes like this: since edge weights are affected by the size of nodes, a filter must be applied to the edges in order to distinguish the significant ones. Following Riccaboni et al. (2013) and Armenter and Koren (2014) we take as as starting point a null model where we assume that edges are randomly assigned to all nodes with probability proportional to their connectivity. The resulting probability distribution is an hypergeometric one. Next we fix a significance threshold and we test if the weights of the actual edges in our layers passes the threshold. If not so, we discard them. The outcome of this procedure is a filtered layer where insignificant edges are removed. This approach is used to standardize the layers using the probabilities created with the filter, solving at the same time both the size bias and the heterogeneity of layers. In a nutshell, we replace

\footnotetext{
${ }^{6}$ Another set of estimates, this time in ten years interval from 1960 to 2000, was also provided in a previous work (Abel 2013).

${ }^{7}$ The others are: exchange of diplomatic representatives, membership in international organizations, armed conflicts and militarized disputes.
} 


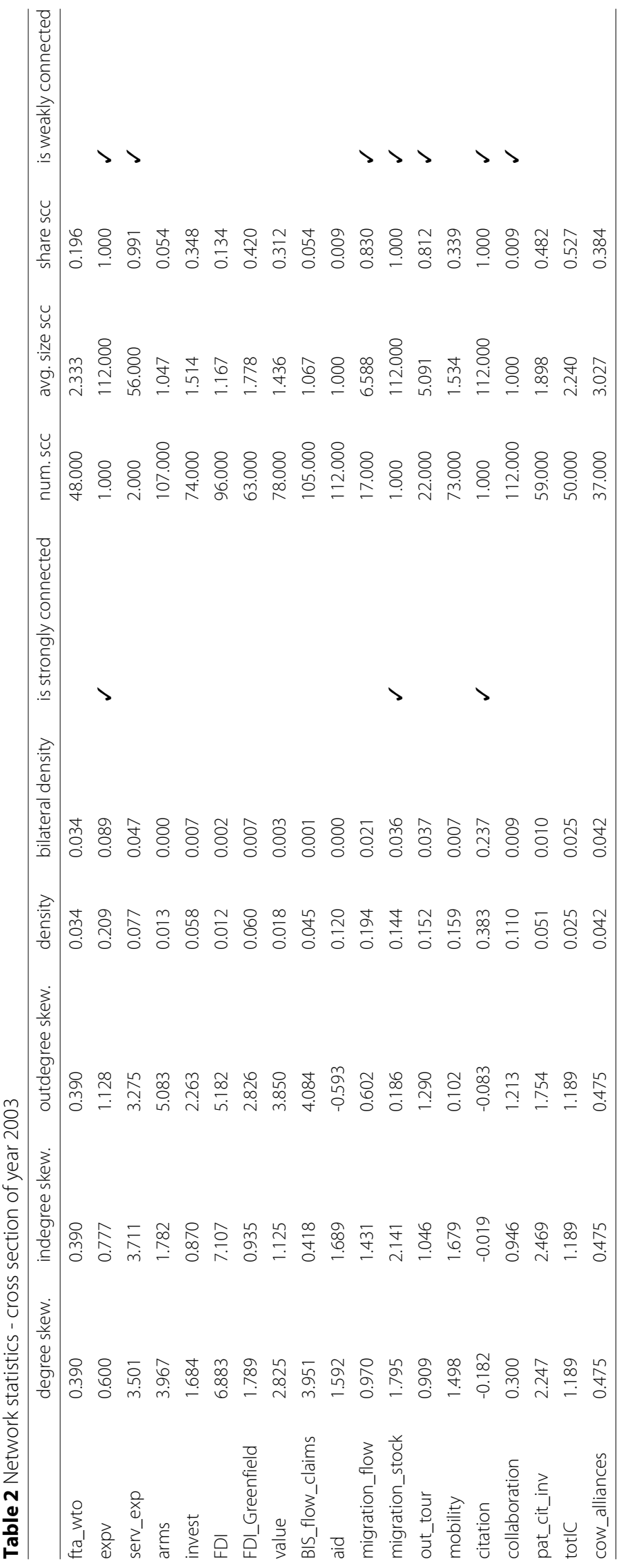




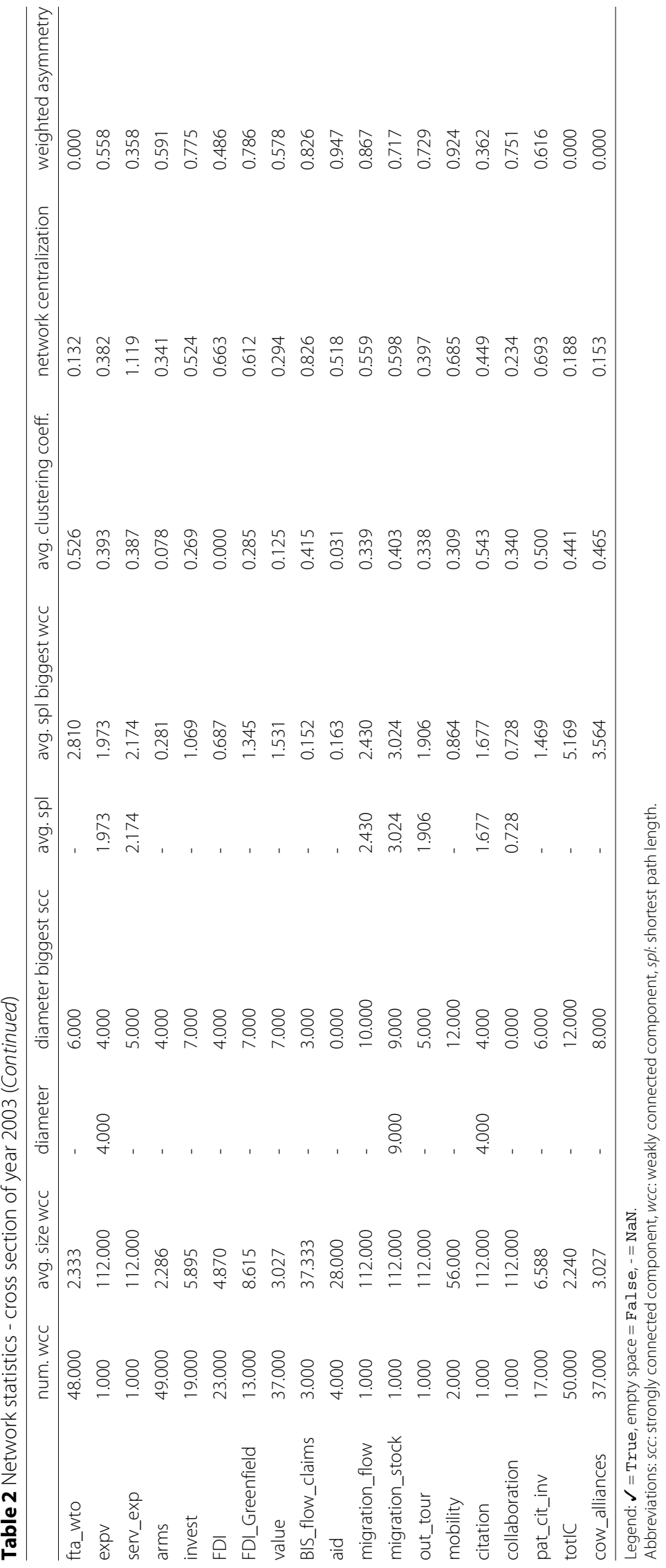


heterogeneous links with the probability of them being more intense than expected under a random null model.

The final effect of the hypergeometric filter is to reduce the density variance by smoothing out denser layers and leaving almost untouched sparse layers. This follows our assumption that more connected nodes (in denser layers) have a greater share of irrelevant edges, while less connected ones have few relevant links which pass the filter. Figures 15 and 16 in the Appendix demonstrate this by showing the amount of observations eliminated by the filter in every layer and how this quantity is positively correlated with the layer density before filtering.

Our choice of the filter was made to reduce the variation in density across layers to make them comparable. However, we have not controlled for variation in other possible features, such as the clustering or the weak-tie structure, which might have been altered as a consequence of our procedure.

Other filters could have been chosen to preserve different characteristics of the networks. Some examples are the disparity filter of (Serrano et al. 2009), the GloSS filter of Radicchi et al. (2011) or the Maximum Entropy approach proposed in Gemmetto et al. (2017). While some of these methods are certainly better to avoid unwanted alterations in the structure of the filtered networks, as demonstrated in Gemmetto et al. (2017), we see two possible difficulties in our case. First, the disparity filter relies on a different theoretical assumption about the null model used to validate the network, a uniform distribution of links, whereas in our case we have assumed an hypergeometric one. Second, in the other two cases, the full information on the network structure is used to construct the null model. Even though this is is principle a desired property of the filter, we cannot rule out some reporting error in our data which could make the above mentioned methods less reliable. In our approach, by using only information on a given country, we avoid spreading the reporting error to the full specification of the null model.

In the next subsections we show some general network statistics calculated on the filtered multiplex using a very conservative filtering threshold. ${ }^{8}$

\section{A comparative analysis of the international networks}

Given that our interest is in the aggregated multiplex and not in the single layers composing it, we have simplified the presentation of the single layer analysis by computing network statistics on the unweighted version of our graphs, without taking into consideration those assortativity and clustering measures which rely on the directionality of edges. We also do not perform a full fledged analysis of similarity of layers, as in Bródka et al. (2018).

Table 2 reports the network statistics for the cross section in 2003 of our multiplex. A similar table is provided in the Appendix for the cross section of year 2010 (Table A2). To help visualizing some properties of layers, we show in Fig. 1 the rank of each layer with respect to each statistic (left hand side plot) and the correlation matrix measured on these rankings (right hand side plot). A lighter (darker) color in the left panel means that the selected layer (y-axis) ranks lower (higher) with respect to the selected statistics ( $\mathrm{x}$ axis). A lighter (darker) color in the right panel means that the two selected layers have lower (higher) correlation with respect to their position in the statistics ranking (i.e. if correlation is high the two layers rank similar in the same network statistic).

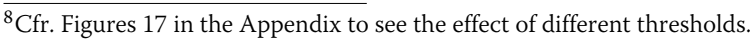


While we see some patterns of similarity, no clear cluster structure emerges: only few layers have a distinct profile (the symmetric layers) while all the others cannot be grouped in a clear manner.

This is evident in plot (a) of Fig. 1: layers rank similarly only regarding some particular statistics, but look very different in all the others. For this reason we do not perform a proper cluster analysis, which will only increase the complexity of the exposition, and we inspect only the macro structure of the layers.

We identify two macro groups of layers with somewhat similar ranking in the statistics. The first one comprehends trade and migration data with the paper citations layer and the three symmetric layers. These last three also form another more compact ensemble in the picture. The other big group is mainly composed by financial layers together with mobility, papers collaboration and patent citation data. While the division in two groups is not clear-cut, it approximately corresponds to the division of layers by typology we introduced at the beginning with layer of different types behaving differently. This suggests that integrating these different data sources may be beneficial.

Moving to the network statistics graph (left hand side of Fig. 1) the picture is more detailed and we can observe differences in the previously identified groups. The first one is the more defined: migration and trade data, together with the citation layer, are usually denser, both in the simple and in the bilateral sense, they have bigger average size and diameter of their bigger strongly connected components, longer average short path length and larger size of their weakly connected components. On the other hand, they have fewer components, their degree (including outdegree and indegree) is usually more skewed to the right and they have lower values of network centralization and weighted asymmetry. These characteristics are also shared by the next three layers (cow_alliances, fta_wto, totIC) which are also less dense.

Other layers exhibit an opposite behavior. There is a set of layers (FDI, arms, value) which is highly anti-correlated with the previous ones: they all rank higher in the left hand

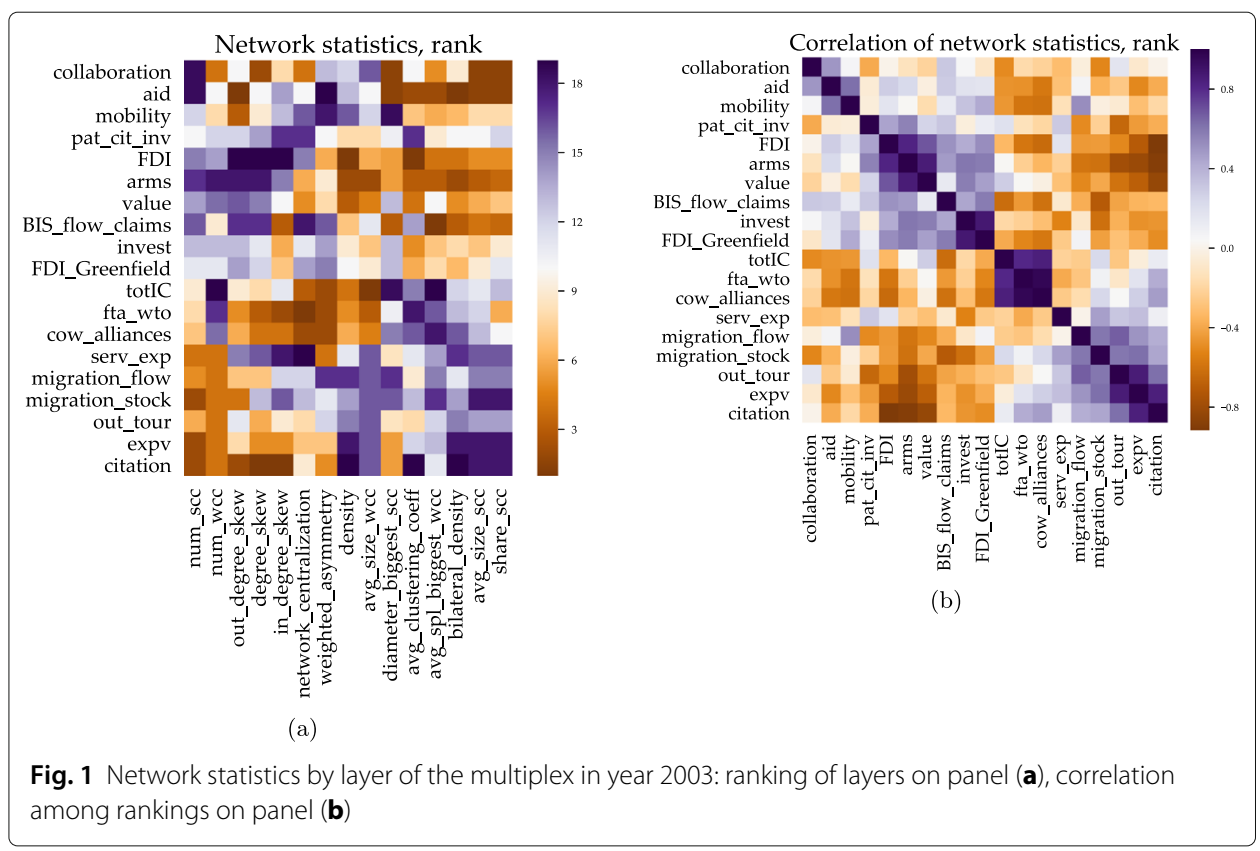


side statistics, i.e they have greater number of weakly and strongly connected components and degrees skewed to the left. Moreover they show lower rankings in the statistics where previous layers showed higher rankings: they are less dense, have lower average clustering coefficient and their biggest weakly connected component is smaller and has lower average short path length. Moreover their largest strongly connected components have smaller diameter and size.

Finally, the remaining layers have less sparkly defined characteristics: while, on average, they have opposite behavior with respect to the initial ones the differences are not so clear. In particular, like the first layers, they have higher density and higher average size of their biggest weakly connected component. However, for the remaining statistics, they differ from the first set of layers.

\section{Multiplex centrality}

We define a multiplex $M$ as a set $L$ of layers, where each layer $l$ is a network defined as two coupled set of nodes $N^{l}$ and edges $E^{l}$ connecting nodes. In our case nodes are countries and edges represent different types of relationships between them. For instance, countries like USA, Russia and India are simultaneously connected by trade, migration and knowledge flows (see Fig. 2). Therefore, two nodes are connected in the multiplex if they have a link in at least one layer.

To exemplify this reasoning in Fig. 2 we can see a selection of nodes and links from the international multiplex: 3 layers have been represented (export, migration and patent citations). For each layer, four nodes have been chosen for each of the 4 quartiles of the GDP per capita distribution (PPP) and placed in ascending order on the vertical axis. The size of the nodes is proportional to their degree in each layer while the color intensity of the nodes corresponds to the centrality of each of them in the whole multiplex calculated using the MultiRank centrality explained in "MultiRank" section. We can see that Uzbekistan (UZB) and Camerun (CMR) are isolated nodes in the patent citations layer, but not in the migration and export ones. Moreover we can see how self-loops are present only in the patent citation layer. In our multiplex there is no link across layers, but the same countries are present in all layers (Kivela et al. 2014).

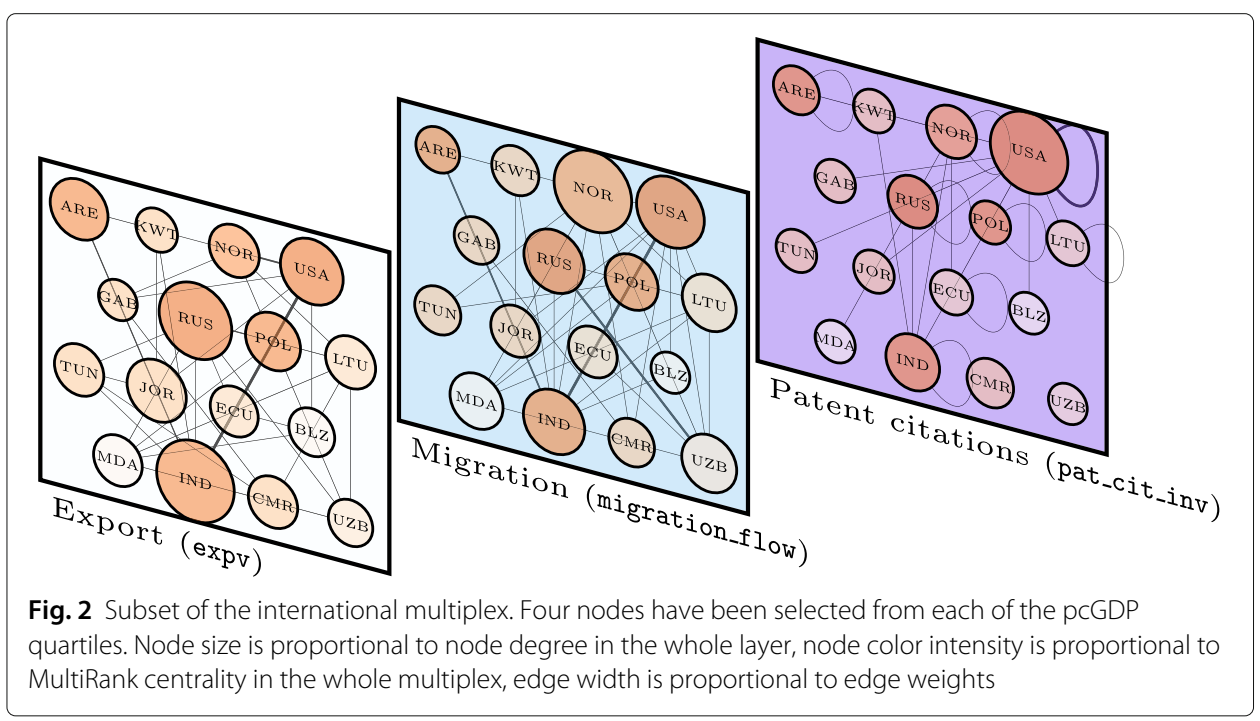


The centrality of a node in a multiplex network is no more a local measure related to its role in each single layer, but becomes a global measure affected by all relationships in which the node takes part. To measure country centrality we have selected two different algorithms which generalize single layer centrality measures to multilayer networks and have the nice property to provide also a ranking of layers as output. This allows us to avoid any a priori assumption of the relevance of layers. ${ }^{9}$

The two algorithms are the multiplex PageRank (MultiRank) (Rahmede et al. 2018) and the multiplex HITS(MD-HITS) (Arrigo and Tudisco 2018), also known as "hubs and authorities" algorithm. In the next subsections we provide a brief description of the two methods, while we refer to the original works for any further details.

\section{MultiRank}

The (single layer) PageRank of a node is a measure of node centrality that accounts for the importance of the node by looking at its centrality and the centrality of all the inneighbours pointing to it.

Formally, given an unweighted network of $N$ nodes, the PageRank of node $i$ can be defined as (cfr. Halu et al. 2013):

$$
x_{i}=d \sum_{j} \frac{A_{i j}}{g_{j}} \cdot x_{j}+\frac{1-d}{N}
$$

Where $d$ is the so called damping factor, $A_{i j}$ is an element of the unweighted adjacency matrix of our network $A$ and $g_{j}$ is the out-degree of node $j$ (i.e the number of its out-neighbors) when its greater than 0,1 otherwise. Hence a random walker will choose among the out-neighbours of node $j$ with probability $d$ and with probability $1-d$ will switch to one of all the other nodes in the network. Starting with a uniform distribution and running iteratively the algorithm we should obtain a stable distribution of the PageRank for all nodes.

When we move from single to multiple layer networks, nodes will have multiple dimensions over which nodes share links, hence the PageRank centrality of nodes is affected both by the single-layer centrality and by the centrality of a layer in the multiplex. Therefore, a rank of the layers of the multiplex is needed to compute the multiplex version of the PageRank.

Following Rahmede et al. (2018) we summarize the multiplex from two perspectives: first as a colored network with links of different colors having different influences, second as a bipartite network of nodes and layers. Then we use both dimensions to obtain a generalized multiplex PageRank. This multilayer version of the PageRank algorithm can be defined for directed and undirected networks as well as weighted and binary networks, hence the adjacency matrix $\mathbf{A}^{\alpha}$ will refer without distinction to any of these types of layers.

From the colored network perspective we obtain matrix $\mathbf{G}$ as the sum of adjacency matrices across $M$ layers weighted by their respective influence $z^{\alpha}$. So, given layers $\alpha=$ $1,2, \ldots M$, the elements of $\mathbf{G}$ are given by:

$$
G_{i j}=\sum_{\alpha=1}^{M} A_{i j}^{\alpha} z^{\alpha}
$$

${ }^{9}$ Obviously, there is still our selection of the layers and nodes in the dataset but, as we have shown, this is related to data availability and comparability. 
From the bipartite view of a multiplex we obtain the $M \times N$ incidence matrices $\mathbf{B}^{i n}$ and $\mathbf{B}^{\text {out }}$ representing the normalized in-strength and out-strength (respectively in-degree and out-degree for binary networks) of each node $i$ in every layer $\alpha$ :

$$
B_{\alpha i}^{i n}=\frac{\sum_{j} A_{j i}^{\alpha}}{\sum_{i=1}^{N} \sum_{j=1}^{N} A_{i j}^{\alpha}}=\frac{\sum_{j} A_{j i}^{\alpha}}{W^{\alpha}} \quad B_{\alpha i}^{o u t}=\frac{\sum_{j} A_{i j}^{\alpha}}{\sum_{i=1}^{N} \sum_{j=1}^{N} A_{i j}^{\alpha}}=\frac{\sum_{j} A_{i j}^{\alpha}}{W^{\alpha}}
$$

Here $W^{\alpha}=\sum_{i=1}^{N} \sum_{j=1}^{N} A_{i j}^{\alpha}$ represents the sum of all edge weights in layer $\alpha$ for directed weighted networks or twice this number if the layer is undirected. For unweighted networks it becomes the total number of link (directed case) or its double (undirected case). Similarly, for undirected multiplex networks $B_{\alpha i}^{i n}$ and $B_{\alpha i}^{\text {out }}$ are identical, since the A matrix is symmetric.

Our specification of the multiplex PageRank works on the assumption that, given a certain node in the multiplex, its centrality will be affected both by the centrality of nodes pointing to it and by the influence of the layers to which these in-neighbors of the node belong. A random walker moves from node $j$ to a neighbour of $i$ along all layers with a probability $\tilde{d}$ and proportionally to $G_{i j}$. Otherwise with probability $1-\tilde{d}$ it jumps randomly on another node of $\mathbf{G}$. The stable distribution we get at the end of this process is the multiplex PageRank centrality of the nodes.

Similarly to the single layer PageRank equation, we get the multilayer PageRank equation:

$$
X_{i}=\tilde{d} \sum_{j=1}^{N} \frac{G_{j i} X_{j}}{k_{j}}+\beta v_{i}
$$

Where $\tilde{d}$ is the damping factor and:

$$
\begin{aligned}
& k_{j}=\max \left(1, \sum_{i=1}^{N} G_{j i}\right) \\
& v_{i}=\theta\left[\sum_{j=1}^{N}\left(G_{i j}+G_{j i}\right)\right] \\
& \beta=\frac{\sum_{j=1}^{N}\left[1-\tilde{d} \theta\left(\sum_{i=1}^{N} G_{j i}\right)\right]}{\sum_{i=1}^{N} v_{i}} X_{j}
\end{aligned}
$$

Here $\theta(\cdot)$ is the Heaviside step function. Equation 4 is a function of the set of layers' influences. To avoid calculating all these values, we couple the above equation with another one aimed at determining the influence of layers. This time we define layerš̌ influence (relevance) as a function of the centrality of their nodes. Hence:

$$
z^{\alpha}=\frac{W^{\alpha}}{\mathcal{N}} \sum_{i=1}^{N} B_{\alpha i}^{i n} X_{i}
$$

Here $\mathcal{N}$ represents a normalization constant. A more flexible specification of this equation can be obtained by introducing some tuning parameters: 


$$
z^{\alpha}=\frac{\left(W^{\alpha}\right)^{a}}{\mathcal{N}}\left[\sum_{i=1}^{N} B_{\alpha i}^{i n}\left(X_{i}\right)^{s \gamma}\right]^{s}
$$

where $a$ regulates the effect of total weight of layers $W^{\alpha}$ on the influence: with $a=1$ layers with higher $W^{\alpha}$ become more influential, while with $a=0$ the layer influence is rescaled with respect to $W^{\alpha}$.

The $s$ parameters instead indicates if more influential layers are those with fewer $(s=$ $-1)$ or more central nodes $(s=1)$.

Finally once $s$ is settled the parameter $\gamma$ allows us to suppress or enhance the contribution of low centrality nodes: with $s=1$ values of $\gamma>1(\gamma<1)$ suppress (enhance) their contribution. Conversely, when $s=-1$ values of $\gamma>1(\gamma<1)$ enhance (suppress) the contribution of less central nodes.

Solving simultaneously the two coupled Eqs. 4 and 9, given a set of parameters $a, s, \gamma$, allows us to assign a centrality $X_{i}$ to each node and an influence $z^{\alpha}$ to each layer $\alpha$ of the multiplex.

Clearly, different choices of parameters will return different rankings. Nevertheless, as we will show in the next section, the configurations can be refined once we require them to be stable enough with respect to the choice of $\gamma$.

\section{MD-HITS}

The (single layer) hubs and authority scores of a set of nodes are two measures of centrality which depend one on the other recursively. For a node to have an high authority score it is necessary to have many high hub nodes pointing to it. Similarly, for a node to have an high hub score it must have a lot of high authority score nodes to point to.

Given the adjacency matrix $A$ of an (unweighted) graph, the hub and authority scores are defined as:

$$
h_{i}=\sum_{j} A_{i j} a_{j} \quad \text { and } \quad a_{i}=\sum_{j} A_{j i} h_{j} \Rightarrow \mathbf{h}=A \mathbf{a} \quad \text { and } \quad \mathbf{a}=A^{T} \mathbf{h}
$$

Usually, the algorithm is calculated by setting all scores to one and iterating for a sufficient number of times, which requires after each step to normalize to one the sum of all the scores.

The generalization of the algorithm for multilayer networks provided by Arrigo and Tudisco (2018) includes layers and time stamps as dimensions to be used to compute the centrality. This results in five scores: two for nodes (hub and centrality scores), two for layers (broadcasting and receiving scores) and one for the time dimension. In our case the last score would not be used since we have only two cross sections.

Similarly to the MultiRank, a node receives an high hub score if it belongs to an high broadcasting layer and has many links towards high authority nodes in layers with high receiving capabilities. Conversely, high authority would be awarded to nodes in high receiving layers with high hub nodes from high broadcasting layers pointing to them.

Finally the definition of the broadcasting and receiving scores of the layers follow when the focus is toward layers instead of nodes.

As for the MultiRank, also the MD-HITS algorithm can be defined on both directed and undirected network as well as weighted and binary networks. Hence, we refer to the adjacency matrix $\mathbf{A}$ without making any distinction among them. 
For each layer $\alpha$ the multilayer generalization of the HITS algorithm reads as:

$$
\begin{aligned}
h_{i}^{\alpha} & =b^{\alpha} \sum_{\alpha} \sum_{j} r^{\alpha} A_{i j}^{\alpha} a_{j}^{\alpha} \\
a_{i}^{\alpha} & =r^{\alpha} \sum_{\alpha} \sum_{j} b^{\alpha} A_{j i}^{\alpha} h_{j}^{\alpha} \\
b^{\alpha} & =\sum_{\alpha} \sum_{j} r^{\alpha} h_{j}^{\alpha} \\
r^{\alpha} & =\sum_{\alpha} \sum_{j} b^{\alpha} a_{j}^{\alpha}
\end{aligned}
$$

where $b$ and $r$ are the broadcasting and receiving scores of each layer. It is straightforward to notice that the overall hub centrality of a node in the multiplex is $h_{i}=\sum_{\alpha} b^{\alpha} a_{i}^{\alpha}$ while its overall authority score is $a_{i}=\sum_{\alpha} r^{\alpha} h_{i}^{\alpha}$. Since we are dealing with a multiplex, our formulation of the layer scores does not allow for some layer to not be connected to others: in other words the inter-layer network in our case is fully connected.

As we can see, there are no parameters to choose in the MD-HITS, hence we will always have one and only one ranking of nodes and layers (according to the score we are interested in). To obtain the centralities of nodes and layers we need to solve the recursive equations defined above, which requires us to solve the eigenvector problem on the whole tensor which represents the multiplex. This can be done by finding an adequate multi-homogeneous map on the adjacency tensor and its unique Perron eigenvector (cfr. Definition 1 in Arrigo and Tudisco, 2018). In Arrigo and Tudisco (2018) the authors then demonstrate the existence, uniqueness and maximality of the MD-HITS measure and provide a converging and fast parallel algorithm to compute it. MD-HITS is an ideal candidate algorithm to compute centrality in a multiplex, since it exists and is unique regardless of connectivity of the graph, while other centrality measures based on eigenvectors require the graph to be strongly connected, a property which is rarely satisfied.

In the next section we analyze the centrality of countries in the international multiplex.

\section{Results}

In this Section we summarize our main results concerning country ranking obtained computing MultiRank and MD-HITS centrality indicators. In particular, we shall focus on two setups regarding the free parameters of the MultiRank algorithm, i.e. $(\alpha, s, \gamma)=(1,1,1)$ and $(\alpha, s, \gamma)=(0,1,1)$. In general, results are fairly robust to alternative specifications, see section "Choice of the MultiRank parameters" in the Appendix for more details. Notice that when $(\alpha, s, \gamma)=(1,1,1)$ we measure the layer importance $z$ without rescaling layers by their weights $(\alpha=1)$ and without giving more importance to layers with more central nodes $(s=1)$. Furthemore, one does not impute more weight on low centrality nodes $(\gamma=1)$.

\section{Preliminary results}

Our dataset contains 19 different layers representing heterogeneous relations among countries. Our objective is to show how different information sources can be integrated in order to obtain synthetic measures of importance of countries and which are the possible differences among these measures. Hence we have focused on 
using the full set of layers at our disposal, discarding unsuitable or repeated layers before constructing the multiplex (more details on this in "Data selection" in the Appendix).

However another possible question would be to check how much of the final results is correlated with the single layer composing our network, i.e. to investigate the redundancy of some layers in determining the multilayer centrality of the nodes. The intuition would be that if layers are heavily correlated among themselves the analysis could be carried on by removing some of them without loosing explanatory power.

Given that this objective is only partially consistent with our focus on analyzing the multiplex as a whole we show here some preliminary results on the correlation among single layer centrality measures of our layers and their multiplex equivalent. It is worth noticing that, however, a clear relation among the multiplex centrality rankings and the single layer ones cannot be found directly given the recursive nature of the multilayer algorithms.

In Fig. 3 we show how much the single layers are correlated among themselves in their centrality rankings and also with respect to the multilayer version of centrality. We can see that the MultiRank algorithm is positively correlated with almost every layer except for two, even though rarely with correlation over $70 \%$. Moreover the layers positively

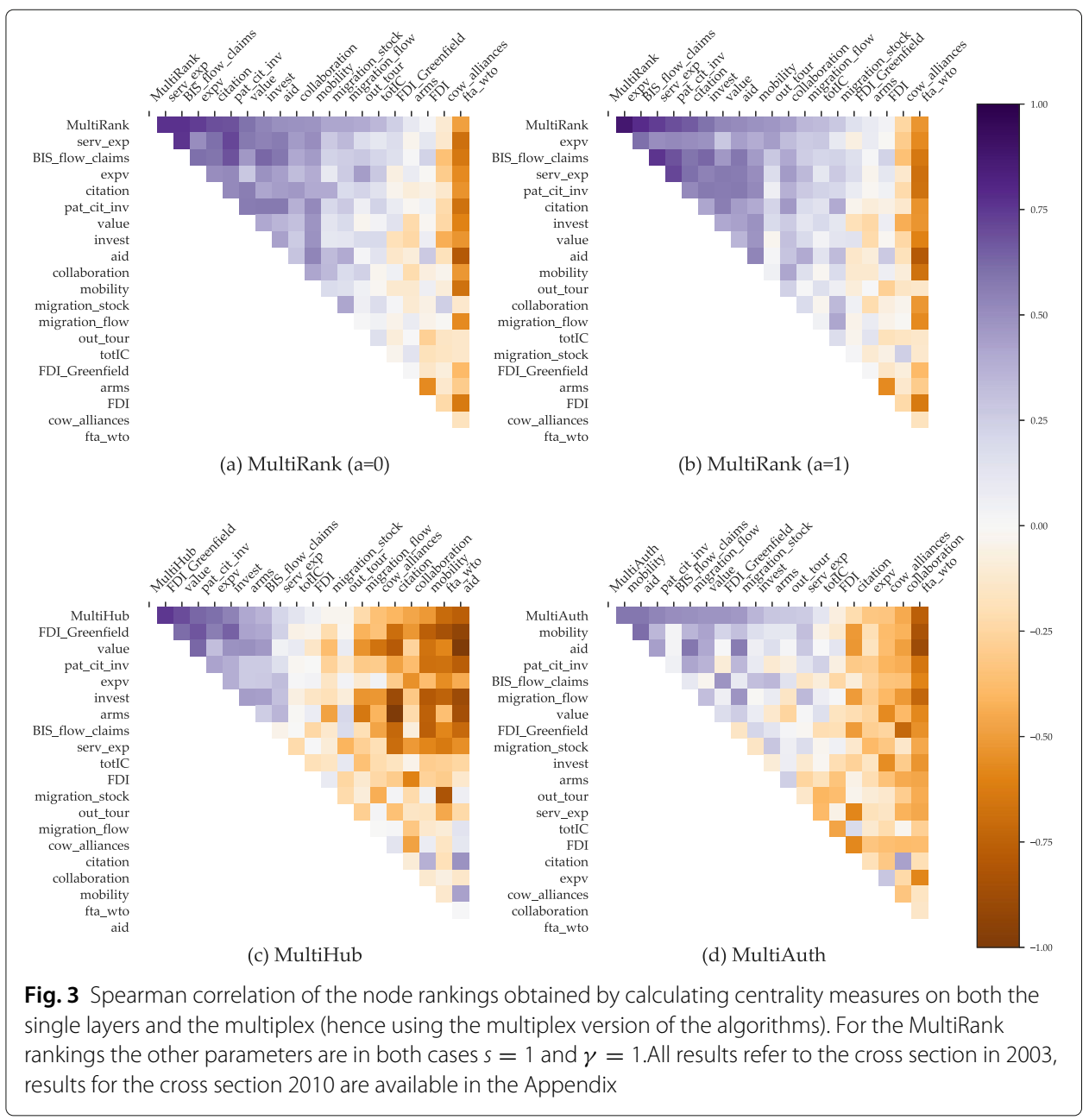


correlated with MultiRank are also correlated among themselves. Conversely for the MD-HITS algorithm the set of layers negatively correlated with the multilayer centrality measures contains more layers. Interestingly the MultiHub and MultiAuth are more correlated with different set of layers hence meaning that removing a set of them or another would affect the centrality measures differently.

In Fig. 4 we have decomposed the set of single layer measures in their principal components and plotted the number of components necessary to explain at least $90 \%$ of the variance in the data (the red step line). We can see that there is some redundancy in the dataset and that the number of required is almost half the one we are analyzing in our work for all the centrality measures. Moreover we have regressed the multilayer centrality measures against the principal components of their single layer versions, added progressively one by one. The blue line in Fig. 4 show how the Mean Squared Error of the regression behaves. We can see that it roughly agrees with the explained variance result and reaches a minimum for a number of component near half the ones we are using (even though with more variability).

All in all, we find that it exists some level of redundancy among the layers in our dataset and that a more parsimonious choice of layers could deliver almost similar results than

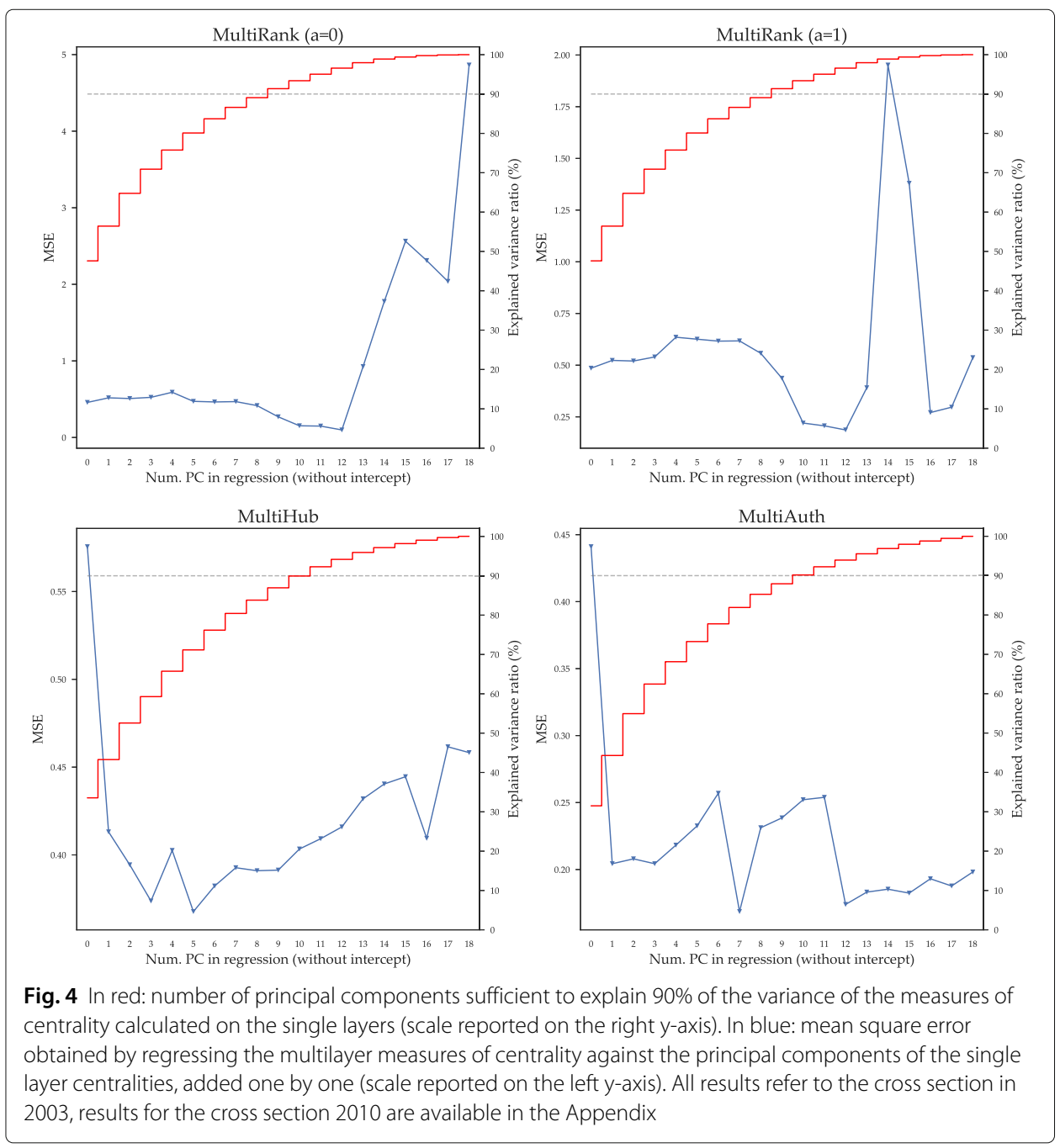




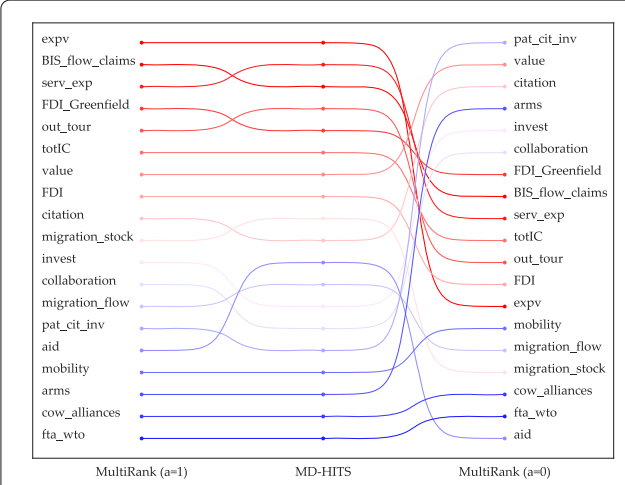

(a)

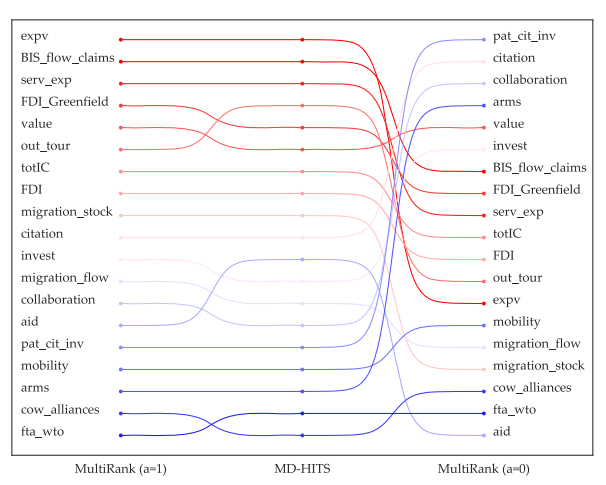

(b)

Fig. 5 Layer rankings in different configurations: cross section 2003 (a), cross section 2010 (b). For the MultiRank rankings the other parameters are in both cases $s=1$ and $\gamma=1$

the one we have made. However how to choose those layers is left to be decided, knowing that some choices could penalize some of the algorithms more than the others. ${ }^{10}$ Given this uncertainty we think our full-fledged approach would at least avoid this type of bias at the cost of more redundancy in the data.

\section{Layer rankings}

The ranking of the layers resulting from the first combination of parameters for the MultiRank is shown in Fig. 5 at the left of the two panels. We can see that this choice of parameters rewards the trade layers (value of exports and service exports), some of the financial layers (BIS loans, FDI flows of both types and the value of M\&As) and some of the mobility layers (temporary migrations and migration stocks). Even though some choices of the algorithm are not obvious (especially among layers of similar kind) one can find a final ranking which fulfills some of the usual expectations on which aspects of a country are more relevant when looking at a global level: exports, foreign direct investment, international M\&A, tourism and stock of migration.

The MD-HITS rankings are reported at the center of Fig. 5. The colouring of the lines helps us see that this new ranking is very similar to the MultiRank one with $a=1$, with some layers switching only few positions (the only exception being international aid).

Finally by looking at the two panels in Fig. 5 we can see that over time the two previous observations hold: the ranking of layers does not change and the two algorithms follow each other.

The second configuration of the MultiRank is reported on the right of Fig. 5. In this setting we leave $s=1$ and $\gamma=1$ but now $a=0$ hence all layers are normalized by their total weight. This corresponds to define the layer importance as $z^{\alpha}=\frac{1}{\mathcal{N}} \sum_{i=1}^{N} B_{\alpha i}^{i n}\left(X_{i}\right)$.

This new configuration reduces the effect of different topological properties among layers and makes them more comparable. The result is that we have a new ranking with stark differences from the other two: now trade and migration layers are less important, loosing positions in favor of other layers such as arms trade and patent citations.

\footnotetext{
${ }^{10}$ Indeed while the number of sufficient principal components is similar across all centrality measures, this does not give us any hint about which of the layers we should remove.
} 


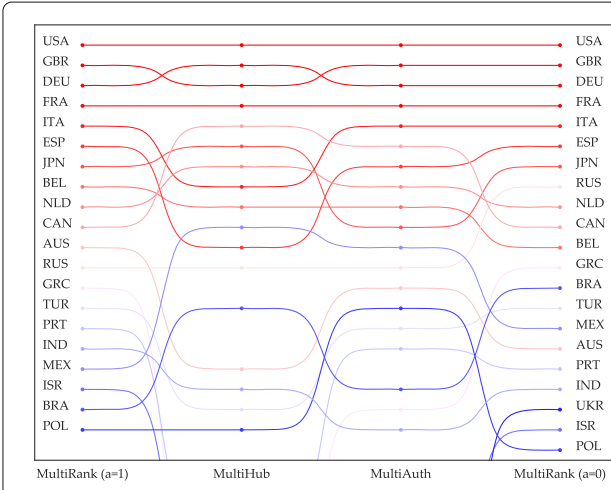

(a)

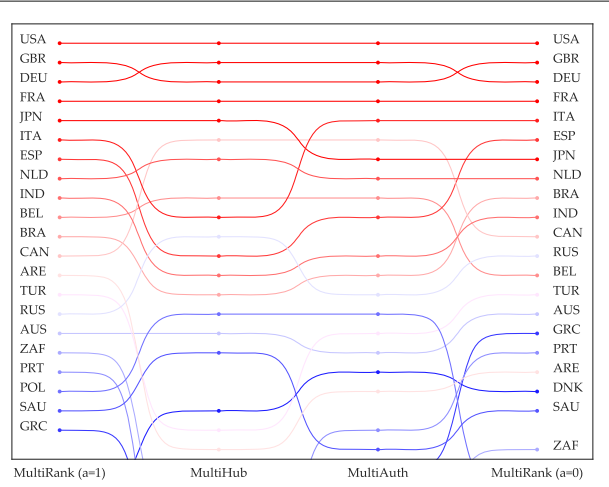

(b)

Fig. 6 Node rankings in different configurations: cross section in 2003 (a), cross section in 2010 (b). For the MultiRank rankings the other parameters are in both cases $s=1$ and $\gamma=1$

\section{Node rankings}

We now move to the comparison of node rankings. Our final goal is to understand how different centrality indicators perform in ranking countries in the multiplex. In Fig. 6 we report rankings for the top 20 countries obtained employing the two parameter setups used for the MultiRank, as well as the multiplex hub and authority scores (MultiHub and MultiAuth).

Results show that, as opposed to the layer rankings, on average the differences in rankings are less pronounced (the blue lines rarely cross the red ones). In fact for the top ten countries we see an almost stable distribution, with countries switching few positions by changing algorithms with the exception of RUS and CAN (this last being severely penalized by the MultiRank and rewarded by MD-HITS) and all algorithms agreeing on the same nodes. In the bottom 10 positions instead we see more differences, especially between the MultiRank and MD-HITS algorithms. In particular the ranking of the multiplex authority score rewards the countries at the bottom at the distribution and penalizes those at the middle. Finally results for the two cross sections show that some countries experience strong catching up or falling behind behaviour. For instance Poland (POL) in the 2010 cross section does belong to the top twenty countries by MultiRank when $a=1$ but with $\mathrm{a}=0$ it loses several positions. On the contrary the United Arab Emirates (ARE) were not in the top twenty countries for any of the algorithms in 2003, while they are in the 2010.

We now ask whether country centrality rankings correlate with country income, as measured by pcGDP. Note that, should one find a perfect correlation, our centrality indicators would not provide any additional insight.

Table 3 shows the Spearman rank correlation coefficient between the nodes ranking resulting from pcGDP and the one resulting from different centrality algorithms. For

Table 3 First two lines: Spearman correlation coefficient between the rankings from pcGDP of the reference year and those obtained by different algorithms. Last line: correlation coefficient between evolution in rankings over time. First 4 columns use multilayer algorithms, last 3 use single layer algorithms on the aggregate network

\begin{tabular}{cccccccc}
\hline & MultiHub & MultiAuth & MultiRank $(a=0)$ & MultiRank $(a=1)$ & PageRank & Hub & Authority \\
\hline pcGDP 2003 & 0.7623 & 0.7607 & 0.6372 & 0.6899 & 0.6745 & 0.7017 & 0.7594 \\
pcGDP 2010 & 0.7779 & 0.748 & 0.6414 & 0.6264 & 0.6873 & 0.7251 & 0.7373 \\
Difference 2003-2010 & 0.31 & 0.137 & -0.1715 & 0.1228 & -0.0772 & 0.3568 & -0.1638 \\
\hline
\end{tabular}


robustness purposes we have added to our four multilayer measures their single layer version (PageRank, Hubs and Authority) calculated on the aggregate multiplex obtained by summing over all the layers the corresponding entries of their adjacency matrices. The first two rows report the rank correlations for our two cross sections, while the third contains the (simple) correlation between the evolution in ranking of pcGDP over time and of the centrality measures. The MultiHub centrality is the one with the highest correlation in ranking with pcGDP consistently between cross sections and over time. It is followed by MultiAuth centrality and the two aggregate multiplex Hub and Authority scores. Both MultiRank indicators perform relatively worse together with the PageRank centrality calculated and the aggregate network. These results show that some of the multilayer centrality algorithms rank countries in a way consistent with the ranking of pcGDP, but with some relevant differences. Specifically for the MD-HITS algorithm they are on average better than their single layer versions calculated on the aggregated network.

Another interesting result is that not only our multilayer measures perform better than their aggregated multiplex analogues, but they also have better performance than single layer centrality measures calculated on each layer. This can be seen in Fig. 7 where we plot the the first two rows of Table 3 as dashed lines and the single layer centrality measures as barplots. We can see that only some layer centralities have a comparable performance with our measures, but none of them is better than our best ones, i.e. MultiHub for both cross sections and MultiAuth for the first only. In the Appendix we are reporting also the geographic distribution of MultiHub, for the cross section of year 2003 and for the evolution of rankings over times (Figs. 21 and Fig. 21).

Finally to further inspect the relation between the node rankings of our algorithms and pcGDP over time, in Fig. 8 we have plotted the change in rank of each country from one cross section to the other for the MultiRank score ( $x$-axis), the MultiAuth score (y-axis), the MultiHub score (coloring of dots) and pcGDP (size of dots). One can observe that while for the pcGDP and MultiHub score rankings we cannot see a clear pattern, for MultiRank and MultiAuth score there is a linear correlation among their rank evolution over time. Moreover this is not affected by the choice of the parameter $a$.

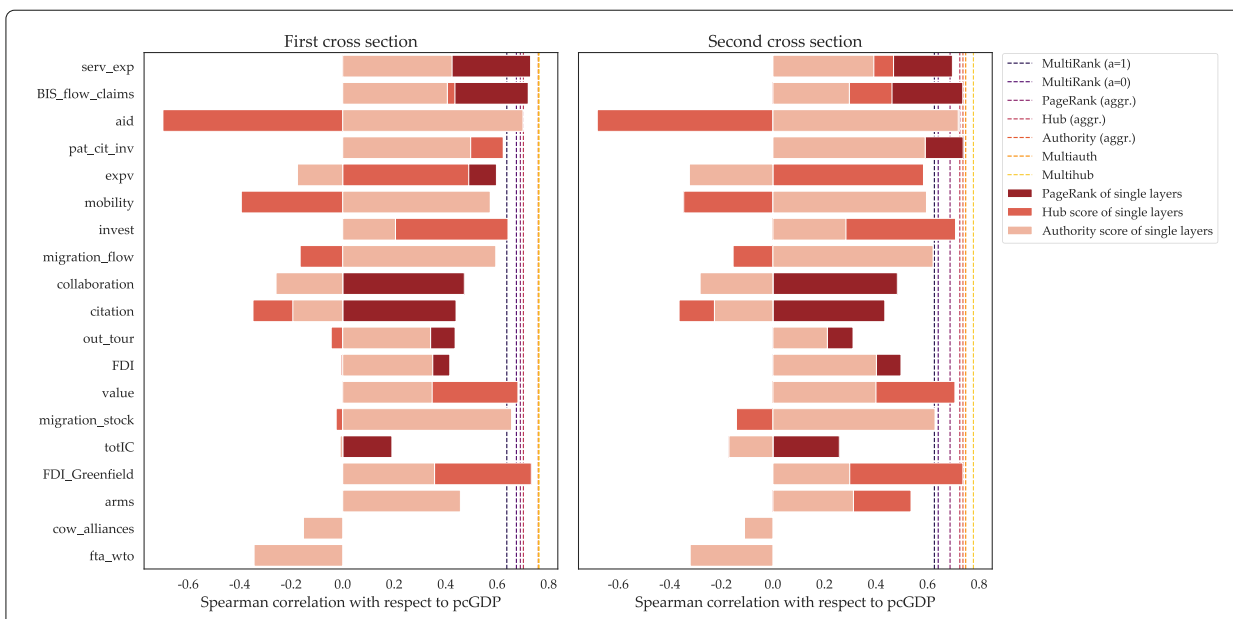

Fig. 7 Performance of single layers centrality measures (stacked barplots) against multiplex ones (dashed lines) evaluated by correlation of their ranking of nodes with respect to pcGDP 


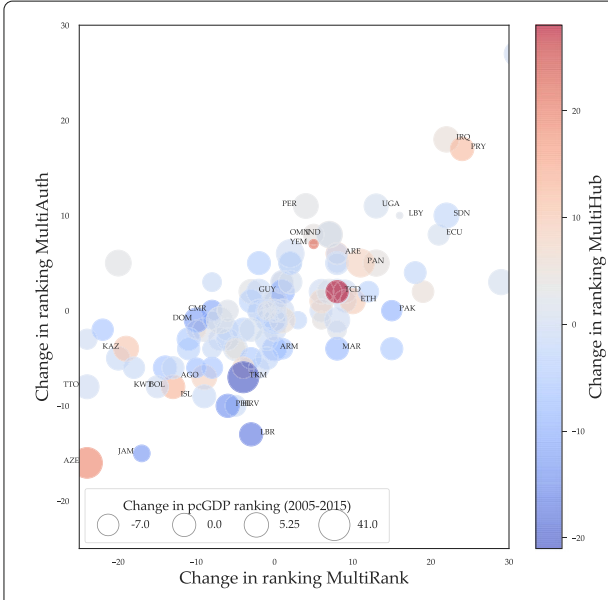

(a)

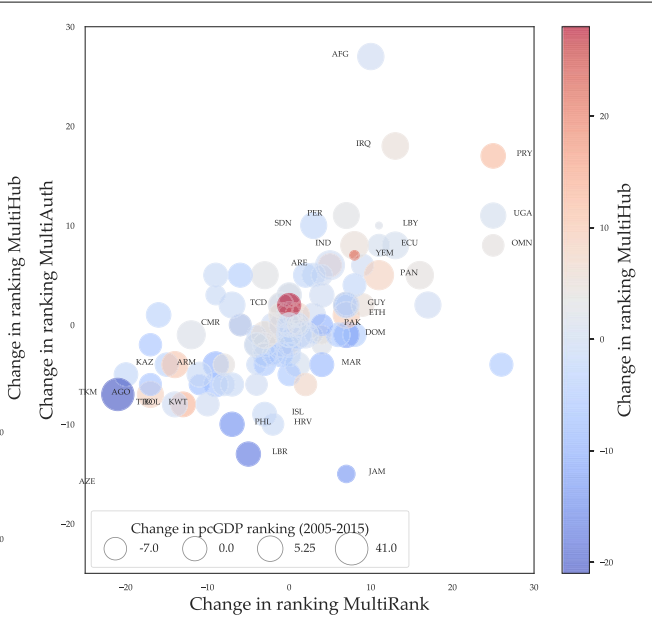

(b)

Fig. 8 Evolution over time of node centrality rankings from different algorithms for $a=1$ a and $a=0 \mathbf{b}$ Labels are provided for the top and bottom 10 nodes in the evolution of the MD-HITS scores

\section{Difference between pcGDP and multiplex rankings}

As mentioned before, similarity with pcGDP is one possible criterion to analyze multiplex centrality. On top of this, one can get additional insights by exploring how centrality rankings deviate from those obtained using pcGDP. Fig. 9 reports the difference in rank with respect to pcGDP ranking in 2003 for the two configurations of MultiRank (Fig 9a) and the two MD-HITS scores (Fig 9b). ${ }^{11}$ Observations at the top of the $y$-axis have greater negative difference between their ranking and the pcGDP one, hence they are being rewarded by pcGDP; while elements at the bottom of the graph have greater positive difference, hence their rank is better according to multiplex centrality while pcGDP ranking penalizes them.

We can see that there are wide differences between the ranking assigned by pcGDP and those assigned by the algorithms: in both cases there are nodes gaining or loosing more than 40 positions in the ranking with respect to pcGDP. Moreover, since the two graphs share the same ordering of rows (given by the Multirank differences), we can see that the rank divergences do not correspond between the MultiRank and the MD-HITS.

To further inspect these differences the behavior of the top ten and bottom ten nodes of the distributions has been analyzed in the inset of the graphs. In the insets we position on the left $y$-axis the ranking by our algorithm and on the right $y$-axis the pcGDP ranking. The "bump" from left to right represents the divergence of rankings with respect of pcGDP, while the thickness of the line represents how much the rankings have changed over time.

Two observations are in order. First, by looking at the insets in the MultiRank graph, the nodes with greater divergence have very different starting MultiRank ranking: we can see that countries being penalized by pcGDP (bottom inset) are coming both from the bottom part of the distribution (Benin, BEN) and from the top part (India, IND). And the same holds in the top inset: see Mongolia (MNG) and Kuwait (KWT).

The second observation is that the same countries evaluated by the MD-HITS algorithm have less extreme behaviour. For instance by looking at the bottom inset of the

\footnotetext{
11 Notice that values have been binned in five element intervals.
} 


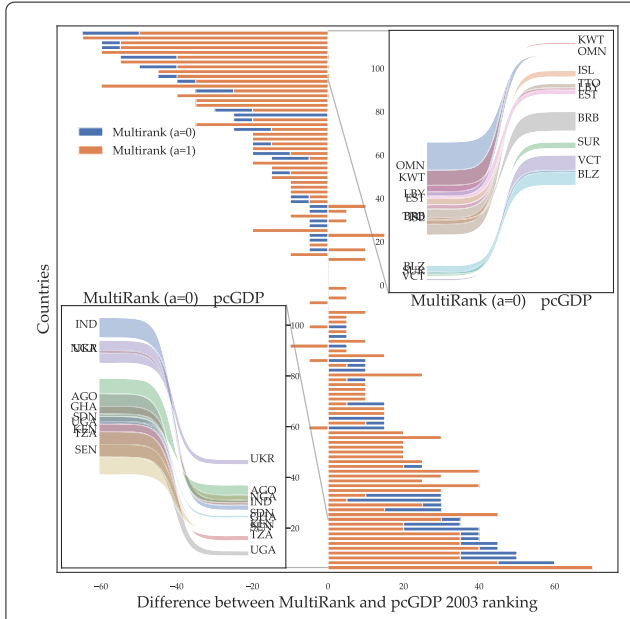

(a)

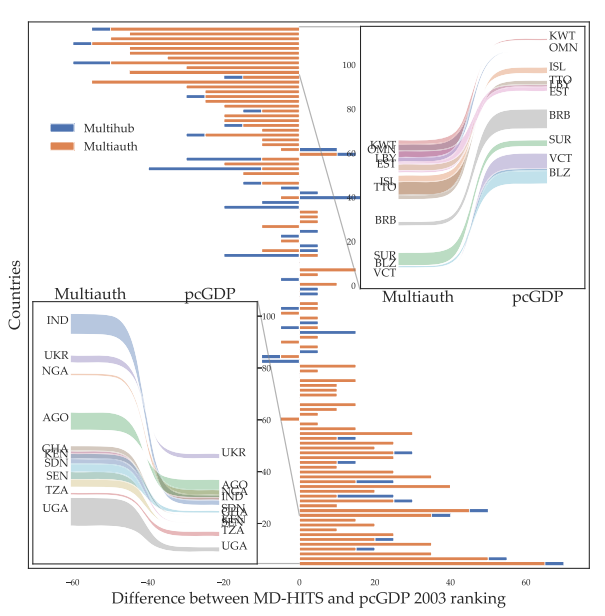

(b)

Fig. 9 Difference between ranking of countries by MultiRank (a) and MD-HITS (b) and by pcGDP in year 2003. The alternative measure for each algorithm is shown in darker color. In the insets only the top and bottom ten countries by rank difference are plotted, with ranking by multiplex centrality on the left and ranking by pcGDP on the right. The width of the line represents the evolution over time of the measures

MD-HITS graph we can see that nodes that were ranked higher in MultiRank with respect to pcGDP, in MD-HITS do not show a great divergence.

Finally in each graph we can also observe with a different color the other ranking obtained by the algorithms: MultiRank with $a=1$ and MultiHub. There are some differences with respect to the principal rankings but less pronounced than the ones between algorithms.

\section{Difference between pcGDP and multiplex rankings over time}

In the last subsection, we have compared rankings obtained using our centrality indicators with the country ranking based on pcGDP. We now explore how country centrality and pcGDP rankings change across the two cross sections. For the sake of simplicity, we focus on countries gaining or losing at least five positions over time in either centrality or pcGDP rankings. We then create four country groups according to whether countries experience a rank improving (worsening) change in the centrality or pcGDP rank.

In Fig. 10 each group of countries has been plotted in a separate graph. The position of the points represents their initial rankings by pcGDP and MultiRank in 2003 while the arrows show the movement in ranking for pcGDP(x-axis) and MultiRank ( $y$-axis) over time. In subplots 2 and 4 their movements in pcGDP and MultiRank are correlated (in direction, not in magnitude) while in the other quadrants they are anticorrelated: while for pcGDP a certain country is expected to gain (lose) positions for MultiRank is the opposite. As a reference to pcGDP growth the size of points is proportional to the compounded growth rate of the pcGDP of the country. Finally Fig. 10a and b show the differences when we change from $a=1$ to $a=0$ in the MultiRank.

The top right (2) and bottom left (4) plots represent those countries experiencing a similar change in centrality and pcGDP. We can see that this is not related to their initial rankings: this happens for Venezuela (VEN), Iraq (IRQ) and Georgia (GEO) in the top graph as well as Mexico (MEX), El Salvador (SLV) and Eritrea (ERI) in the bottom graph which are in different parts of the distribution. At the 


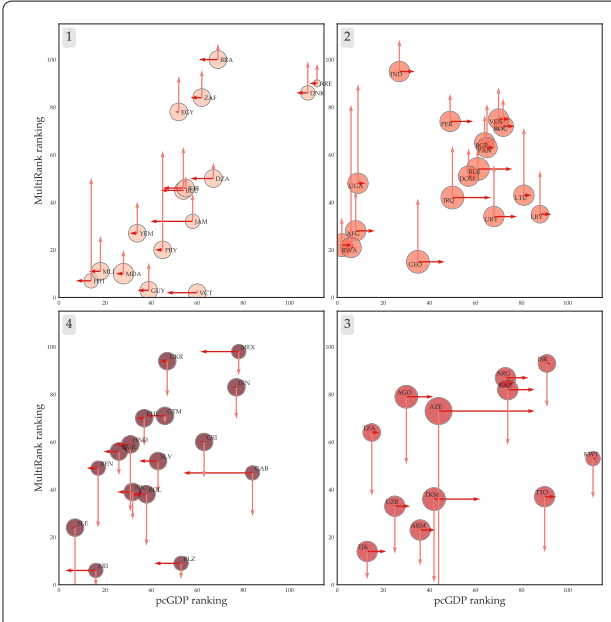

(a)

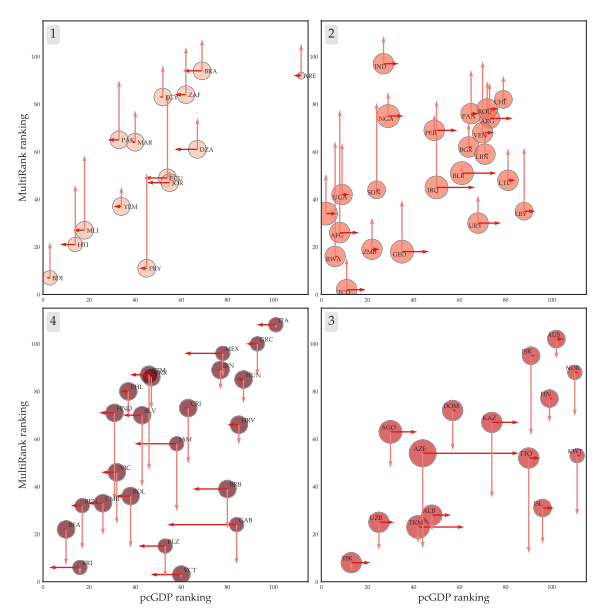

(b)

Fig. 10 Evolution of ranking over time of pcGDP (x-axis) and MultiRank (y-axis). MultiRank calculated with $a=1$ in (a) and with $a=0$ in (b). Countries have been split by the direction of evolution of MultiRank and pcGDP; starting from the top left corner clockwise: 1) increase/decrease, 2) increase/increase, 3)

decrease/increase, 4) decrease/decrease

same time, we notice that in the top right and bottom left panels nodes tend to be distributed along the bisector (hence their initial rankings among pcGDP and MultiRank coincided as well), but with some notable exceptions such as India (IND) and Gabon (GAB).

The top left (1) and bottom right (3) plots instead show countries whose evolution over time did not coincide (in direction) by MultiRank and pcGDP. Top left countries have lost positions by pcGDP but gained by MultiRank, while the contrary happens to bottom right countries. We can see that affected countries have initial position either at the top of the graph (Brasil (BRA) in plot (1) and Israel (ISR) in plot (3)) or at the bottom part of the distribution (Haiti (HTI) in plot (1) and Tajikistan (TJK) in plot (3)) with less countries in the middle of the distibution.

But the most interesting findings come from the difference between plot Fig. 10a with MultiRank using $a=0$ and plot Fig. 10b where MultiRank is calculated using $a=1$.

These differences show that the choice of the MultiRank parameter $a$ and the resulting ranking of layers has an effect on the final ranking of nodes and on their expected evolution. So if we assume that layers have to be rewarded for their total weight $(a=1)$, we get a ranking of layers which rewards trade layers the most (cfr. Fig. 5) and this in turn affects the ranking of countries and affects which of them seem to gain positions over their pcGDP rankings. If instead we prefer to normalize layers by their weight $(a=0)$ we get a different ranking of layers.

While the relation between the choice of parameters, layer and node rankings is clear for MultiRank, we do not have similar insights for the MD-HITS algorithm: we have a unique ranking of layers and two ranking of nodes which take into consideration two different aspects of country centrality.

To have a clear overview on how differently the algorithms we used classify nodes with respect to their rank evolution in Fig. 11 the four quadrants of plot Fig. 10 have been assigned four numerical codes and for each of our algorithms nodes have been sorted in 


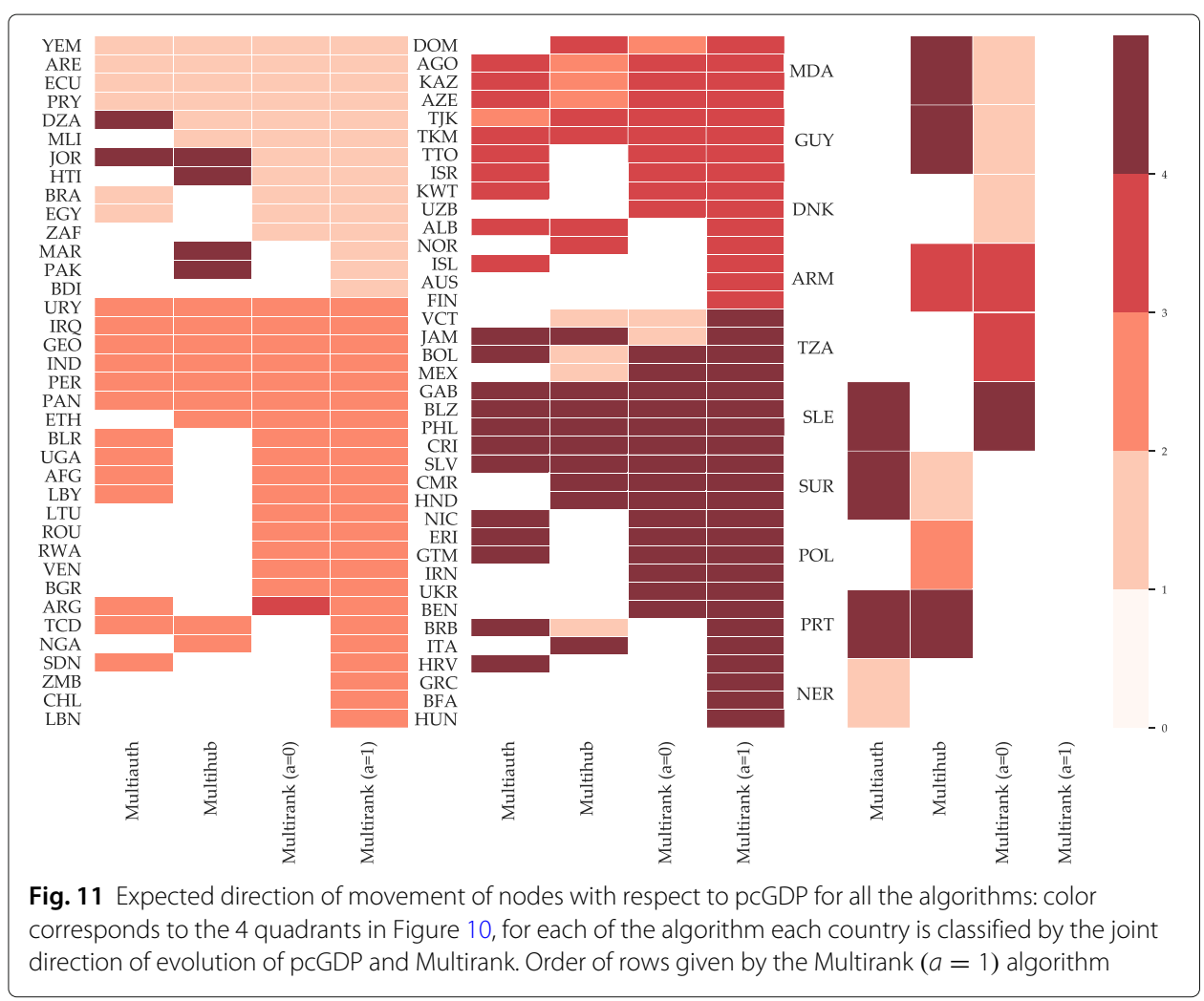

their respective categories. ${ }^{12}$ We can see that there are few nodes where all algorithms agree on the classification, a bigger set where most of them agree and finally for more than half nodes the algorithms provide wildly different classifications.

So if we want to use a very conservative criterion and think that the agreement of the all four algorithms represent a good way to identify some stylized facts, we get the following picture: while by their pcGDP ranking Yemen (YEM) United Arab Emirates (ARE), Ecuador (ECU) and Paraguay (PRI) have not been growing, by their multiplex ranking they have been becoming more central. Another way to see this is that all four algorithm would have placed these countries in panel 1 (top left) of Fig. 11. On the opposite all algorithms agree to place only Turkmenistan (TKM) in panel 3, which identifies countries which have become less central than what their pcGDP growth would have predicted. Furthermore in panel 2 (both the rankings resulting by pcGDP and by multiplex centrality have positive growth) the algorithms would place Uruguay (URY), Iraq (IRQ), Georgia(GEO), India (IND), Perù (PER) and Panama (PAN). Finally in panel 4 (negative growth of rankings) they would place Gabon (GAB), Belize (BLZ), Philippines (PHL), Costa Rica (CRI) and El Salvador (SLV).

\section{Conclusions}

In this work we have collected a new dataset of international network measures, measuring the connections between countries over different financial and non-financial dimensions and using multilayer network analysis for the first time.

${ }^{12}$ Hence to see which countries are "appearing" in the plots, as we discussed in the previous paragraphs, it is sufficient to check the columns where one country is present for one algorithm and not for another (hence the third and the fourth column in our case) 
We have computed different centrality measures and we have shown how different aspects of the data can be highlighted with little change in the parameters of the algorithms and how the resulting change in ranking can be interpreted. Next, we have compared the ranking of countries obtained by network measures and a common measure of macroeconomic performance, such as pcGDP. We have found a satisfying correlation for some of the algorithms, the MultiHub score especially, both at the static cross section level and for the change in pcGDP.

To show the differences between network measures and pcGDP, we have analyzed how each country development trajectory compares to changes in network centrality. We have found only few countries for which all measures agree, while for most of countries different possible trajectories were drawn, not always consistent to those measured by pcGDP.

This may reflect the fact that the development of countries is not characterized by a single path and multiple factors concur to define their growth trajectories, hence new measures are required to capture those differences. However we are conscious that any index that would try to summarize all these aspects over one dimension is by definition an approximation and entails a loss of information.

This work is a first step in order to provide meaningful multiplex measures for the centrality of countries, hence we have left many issues open for future work. We have not addressed any issue of community detection of nodes and layer similarity. Moreover we have not addressed any of the issues of causality over the network nor network reconstruction which we think are too broad topics to be discussed here. Further work will also be required to integrate other sources in the dataset of relevant international relationships.

\section{Appendix \\ Data selection}

Reporting agencies collect data on a fixed set of countries in different years. Hence an empty report would not imply the absence of the country from the dataset but for a given year only. To translate in network theory terms: there may be some nodes in a network whose edge weights with respect to all other nodes are 0 at a certain time $t_{i}$, but are still present in the network with positive edge weights at other $t_{j}$ with $j \neq i$. These nodes belong to the network and we call them isolated nodes at time $t_{i}$. On the other hand, missing nodes don't have link to other nodes at any $t$.

To tell if the absence of a certain node from a specific year means its absence in the overall layer we needed to define when a node is part of our dataset. To do that we have made the following assumption: if a node is present as origin or destination at least once in the whole reported set of years for a certain layer, that node has to be considered as existing in the dataset. ${ }^{13}$ To calculate the set of existing nodes in each layer we have used the whole span of its observations in our dataset, hence not only years 2003 and 2010.

Finally, to get a constant set of nodes across all the multiplex, the existing nodes of each layer must match across all the others. The result of this process is a set of 112 nodes common to all our layers. By referring to the nodes column in Table 1 we can get

$\overline{{ }^{13} \mathrm{~A} \text { more strict assumption would }}$ be to use only nodes existing at least once as sources. 
a good approximation of this process: we define the set of nodes for each layer as the union of the existing nodes in the years 2003 and 2010. Hence their size corresponds to the maximum number between $t 0$ and $t 1$ in Table 1 and ranges from 211 in the free trade agreement layer to 124 in the alliances layer. This leaves us with 19 different sets of nodes, one for each layer, partially overlapping. To obtain a common set of countries for the whole multiplex we calculate the subset of all the individual layers' node sets. Hence our final set is a group of 119 countries and the difference between 124 and 119 is given by five countries belonging only to the alliances layer and not to the rest of the multiplex, which we have removed.

It must be noted that this process leads automatically to select the smallest set of common countries across all layers. To avoid reducing this number too much we had to select a minimum threshold of countries to include in the multiplex with the consequence of eliminating some layers which would have had observations in the required years but with a reduced number of nodes (Fig. 12 in the Appendix show some of the layers we have eliminated with this criterion).

\section{Data preprocessing}

Our final result is a multiplex of 19 layers with 112 nodes in each of them, observed at two "rugged" time snapshots, i.e. with not exactly the same starting and ending year for all layers. Balancing our two cross sections on the same selection of nodes and layers ensures that we can make meaningful comparisons over time, but some further steps are required before moving on to the actual analysis.

First we have preprocessed our data to remove inconsistent observations. While in principle network weights do not have strict requirements to satisfy, in our case we needed to impose some restrictions: since our network layers are mostly directed, negative weights

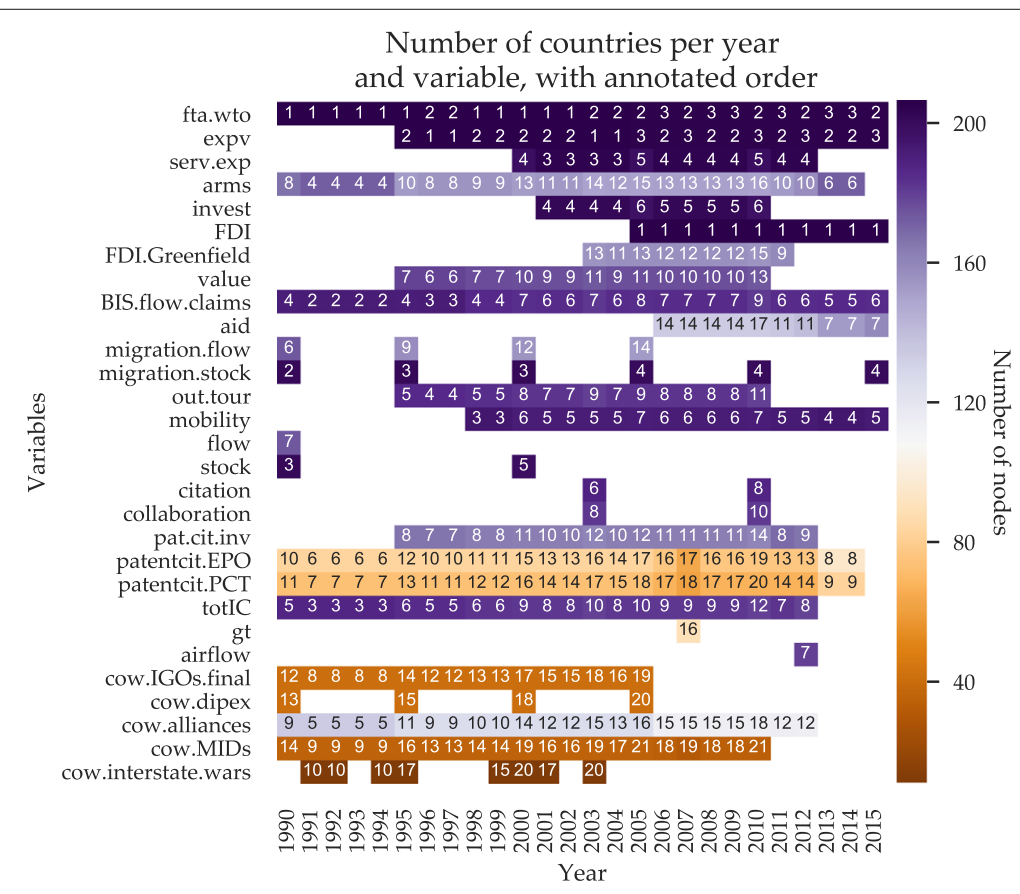

Fig. 12 Overview of the full dataset 


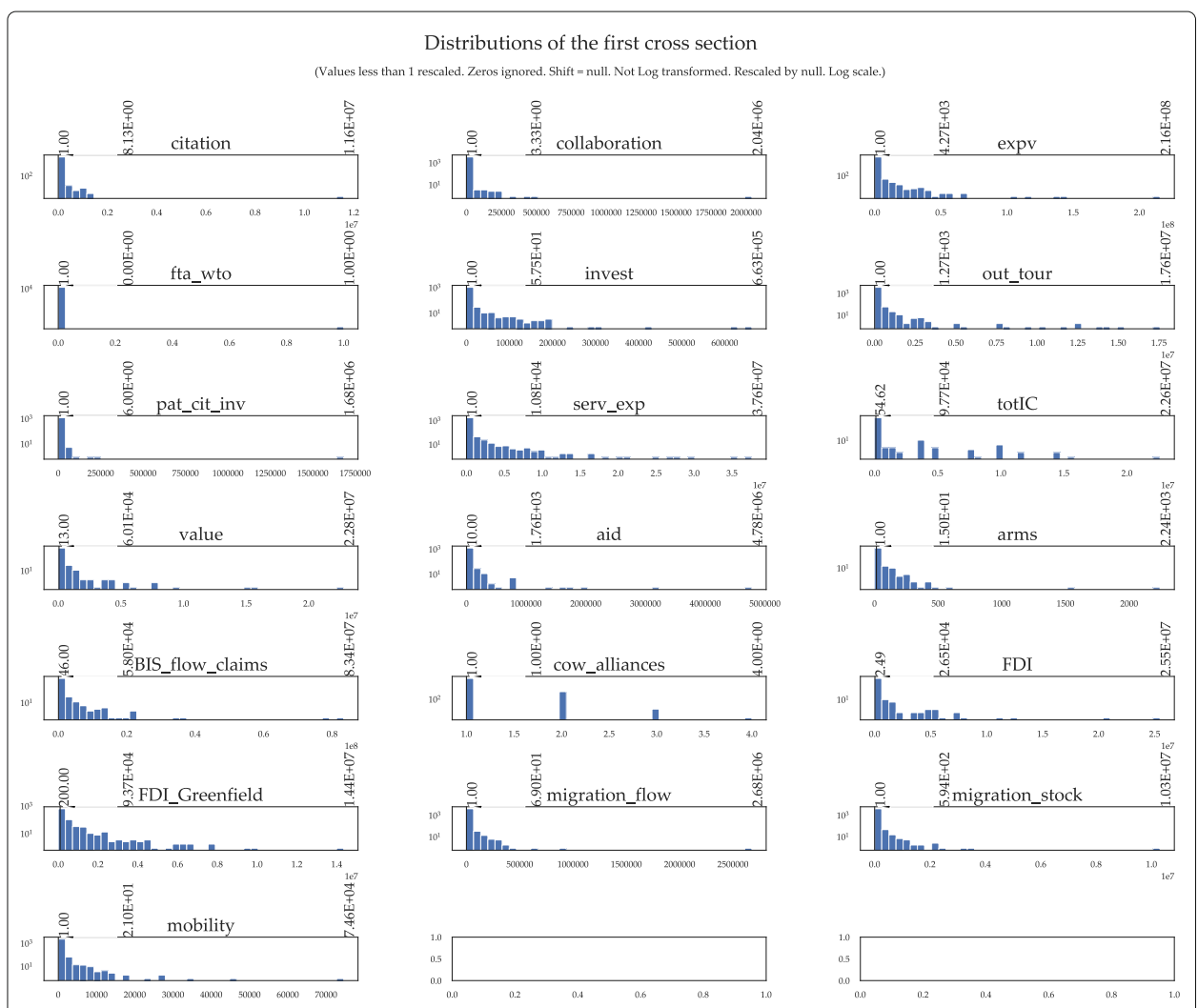

Fig. 13 Positive weights distribution in each layer of the cross section in 2003. Values rescaled to 1 if lower than 1. Logarithmic scale on the $y$ axis

have no meaningful interpretation. They should instead be rewritten as positive edges in the reverse direction: if country $A$ receives a negative flow from country $B$, it actually means that country $B$ is the source of a positive flow towards $A$. Hence we have reversed negative flows when possible, i.e when the whole layer showed a consistent pattern of reversed flows and there were no duplicates after reversion. If instead most negative weights seemed randomly distributed or reverse flows were already recorded in the dataset we have dismissed the negative values as computational errors. ${ }^{14}$

Another type of requirements we imposed is to have edge weights not smaller than one, in order to avoid problems with eventual filtering operations. As one can see from the minimum weight column in Table A1 several layers have fractional values. Most of the time this was due to the unit of scale employed, hence it has been sufficient to rescale them with the right multiplier. This is the case for FDI, FDI_Greenfield, BIS_flow_claims, aid, value, serv_exp and totIC layers.

The other cases of fractional weights are the results of citations of multi-authored works which are attributed to certain countries only with the fraction related to a specific author. To deal with this we have added by default a single citation to all the fractional citations ${ }^{15}$.

The last restriction we have imposed on our data regards the way we intend to interpret our results. Since the basic stylized fact we want to recover from the MultiRank and

\footnotetext{
${ }^{14}$ This issue affected a small number of layers with marginal effects. Only in one case, the BIS layer, the share of negative values was substantial (around 15\%).

${ }^{15}$ Another option would have been to rescale all the citations by the minimum, but this would have made the whole distribution of weights explode.
} 
Bonaccorsi et al. Applied Network Science

(2019) 4:126

Page 29 of 42
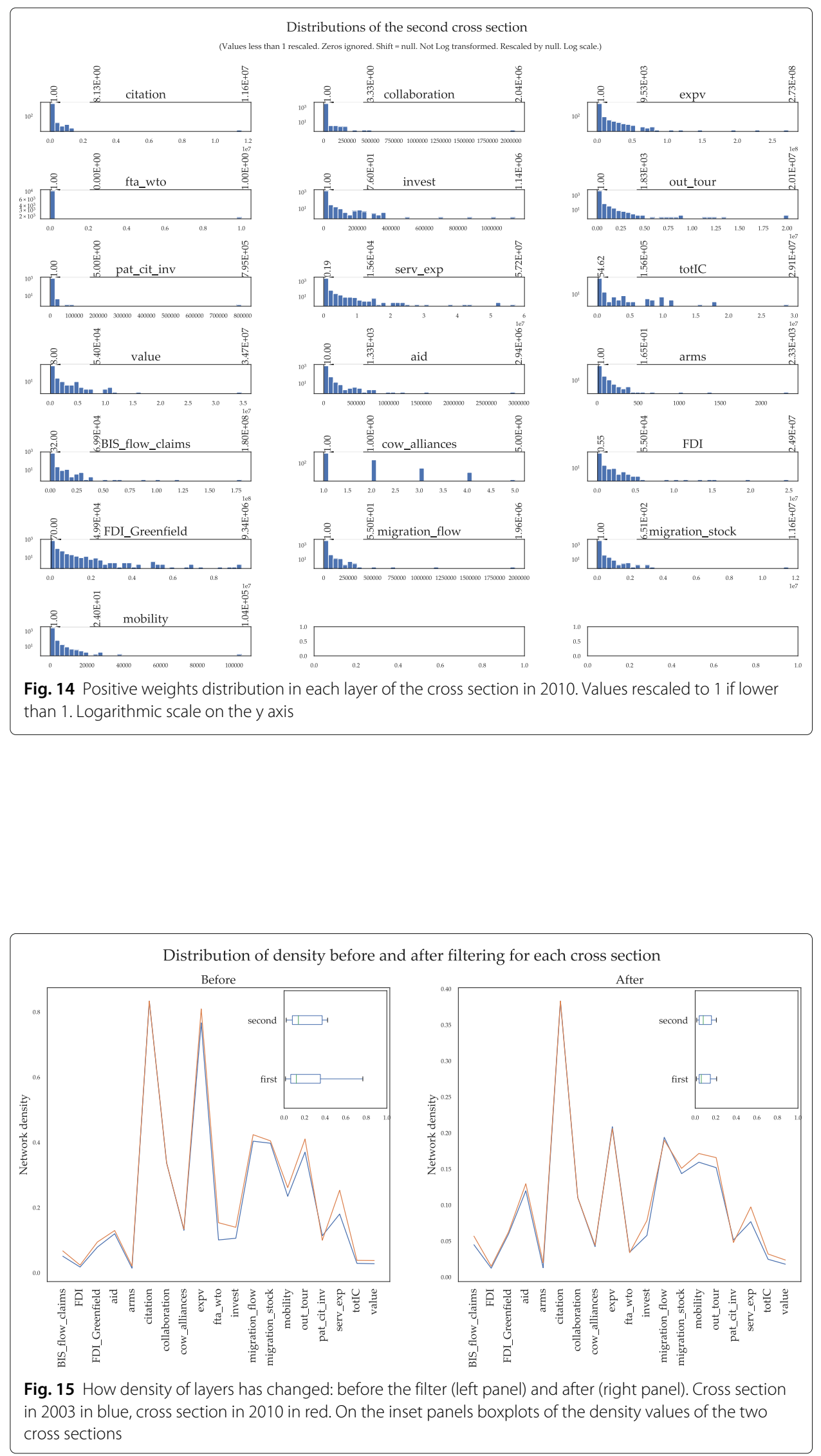


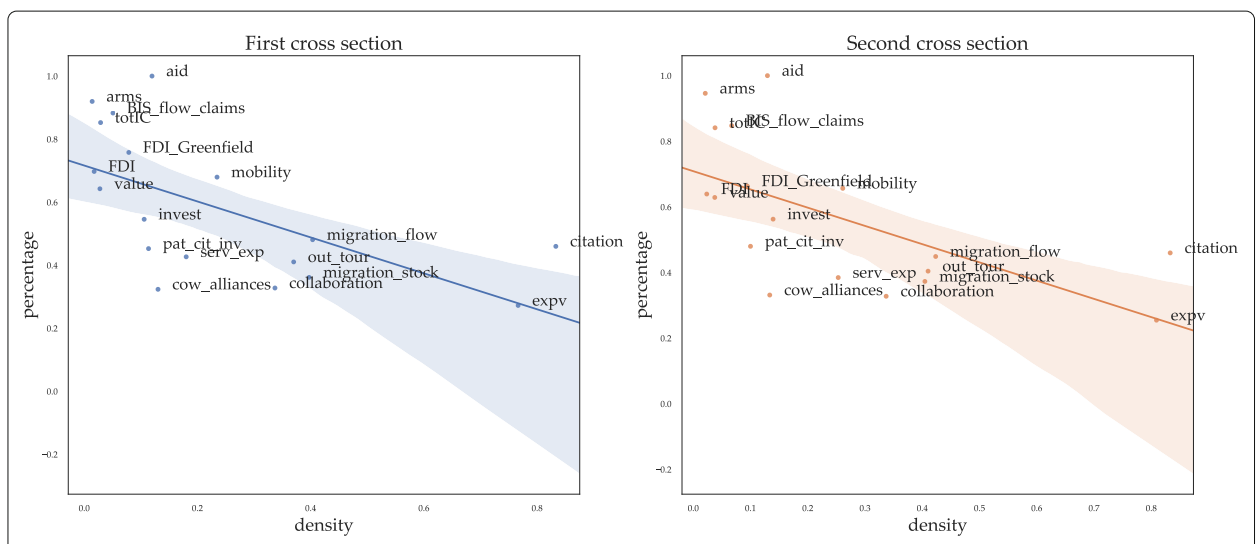

Fig. 16 Relation between percentage of values preserved by filter and density before filtering

MD-HITS algorithms is the division of countries in two groups, developed and underdeveloped countries (a north-south view of the word), we need our layers to be oriented in the right way: the concentration of flows toward some countries must reflect their centrality as developed nodes in the network. To achieve this we have inverted the international aid layer, as we said before, and the migration stock one which, by construction, tells us how many persons from the source country migrated to the destination one. On the contrary in a more north-centric view of the world we will need to know how many migrants the source country hosted from the destination one, since migration flows usually points from south countries to north ones.

\section{Choice of the multiRank parameters}

Results of the MultiRank algorithm with respect to changes in each of the three parameters $a, s, \gamma$ (for nodes only the top 18 elements are shown) are reported in Fig. 18a and b. We can see that choosing a different combination of parameters alters the final ranking of nodes and layers and that some combinations experience more abrupt changes than others. While for the MD-HITS we don't need to provide any parameters in order to obtain the final rankings, for the MultiRank we need to choose how to specify them. In what follows we will show how we choose our two configurations.

The process which led us to the choice of the final configurations of the MultiRank starts by making sensible assumptions on how the ranking of layers should be performed: as we already explained in the main text the safest configuration is $(1,1,1)$ where the content of the layers is considered as it is, without making any adjustments. The next best candidate is the configuration where $a=0$, hence where we try to adjust for differences in the total weight of layers, which we have shown to be non negligible even after filtering, still without making any other assumptions on the importance of central nodes inside each layer, which will require us to introduce further assumptions on the data.

In Fig. 5 one can see the two rankings of layers which are the results of the previous assumptions. Now the second step in the evaluation of the two configurations is to move to the node rankings deriving from them and compare their stability with respect to the parameter $\gamma$ which from Fig. 18 seems to create disruptions in the rankings even when keeping fixed the other two parameters. We would like to have two configurations of the 


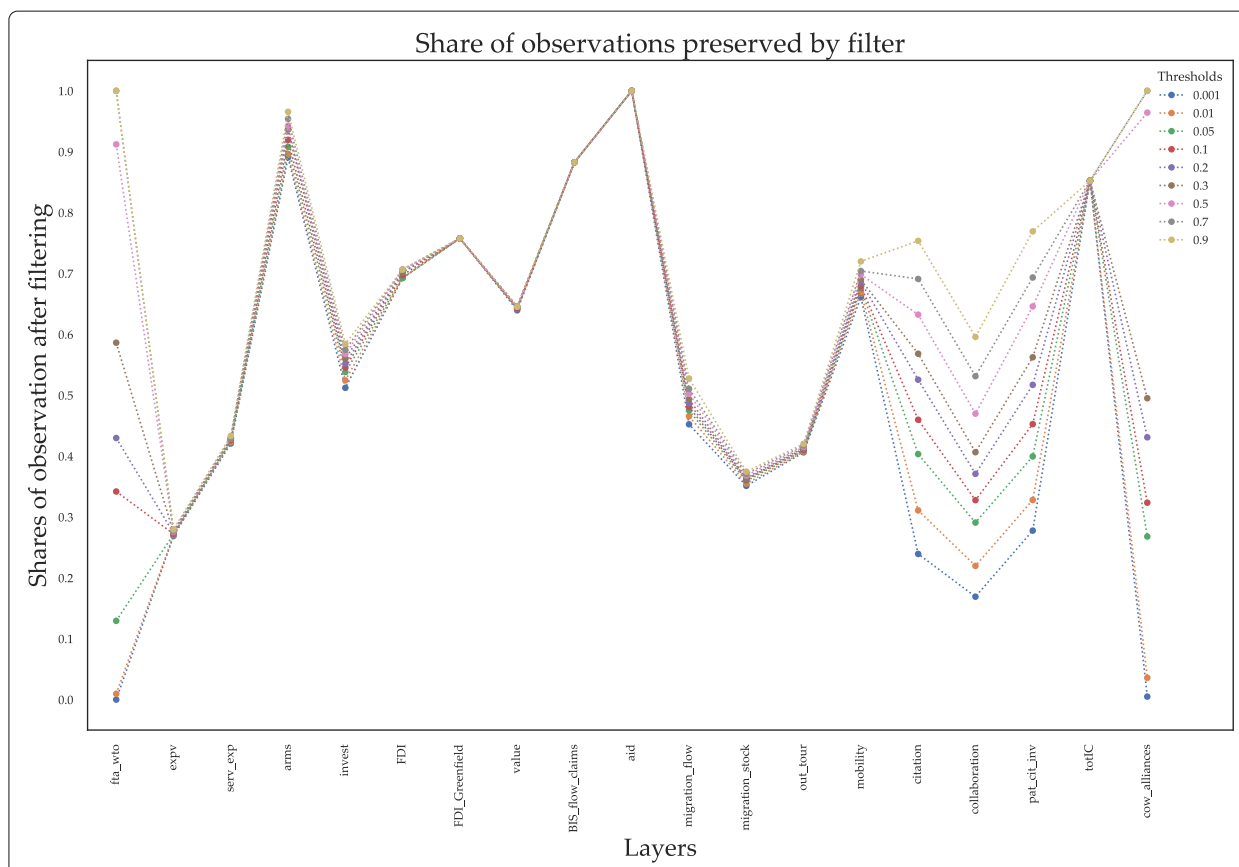

(a)

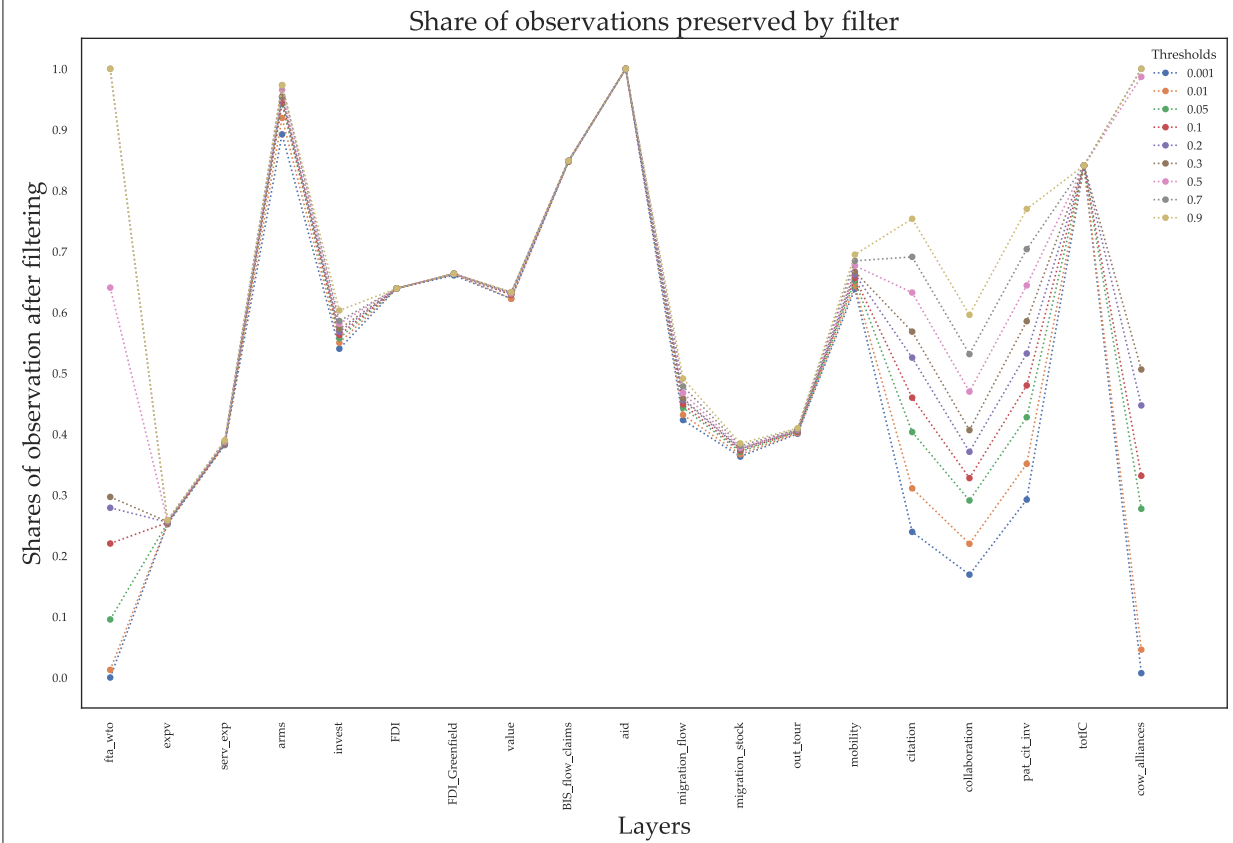

(b)

Fig. 17 Sensitivity of the preserved values after filtering with respect to the change of the threshold of the filter. Cross section in 2003 (a) and in 2010 (b)

MultiRank which are not heavily affected by the choice of $\gamma$ in at least the majority of the range of its values.

Finally the third criterion to evaluate our choice of parameters is by comparing the similarity of the node rankings with respect to the one stemming from pcGDP. We would like our choice of parameters to have a good correlation (in rank) with pcGDP which will mean that our centrality measures are capturing a good signal from the underlying 


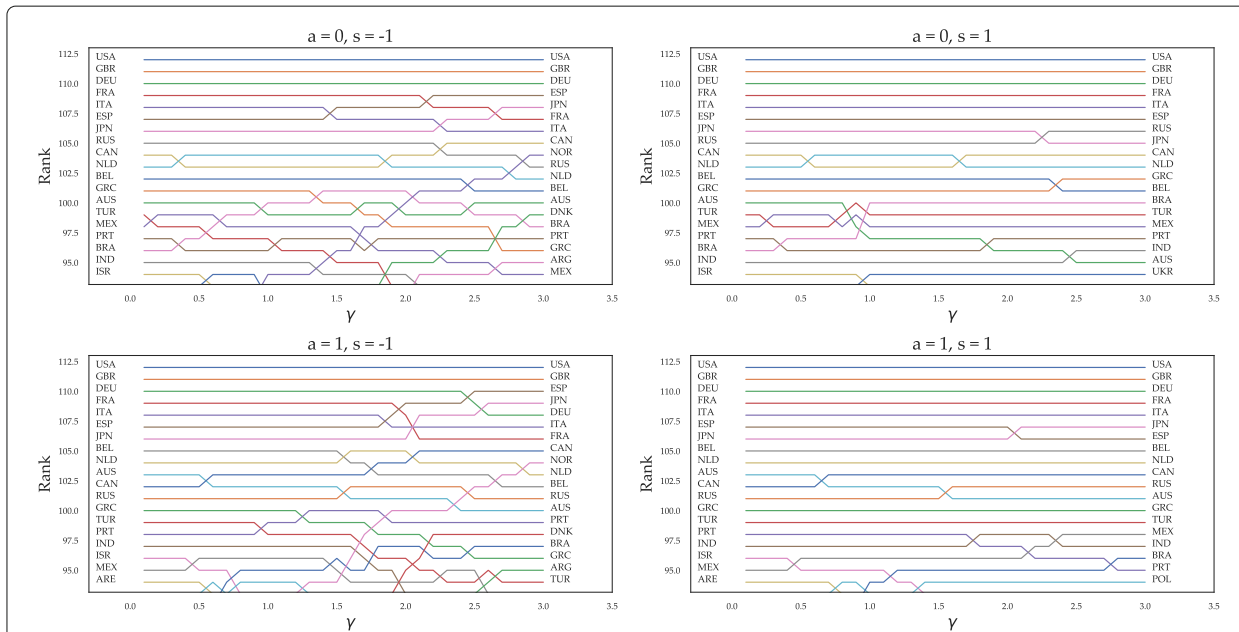

(a)
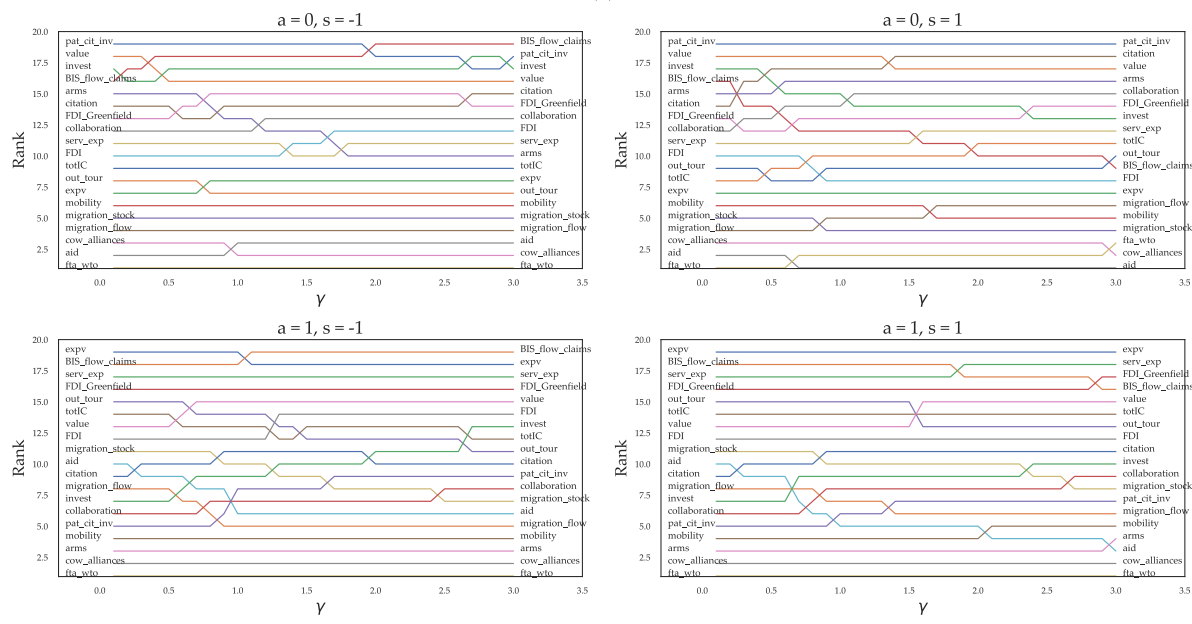

(b)

Fig. 18 Evolution of MultiRank for the 18 top countries $(\mathbf{a})$ and for all layers (b) in the dataset with respect to different parameters choice
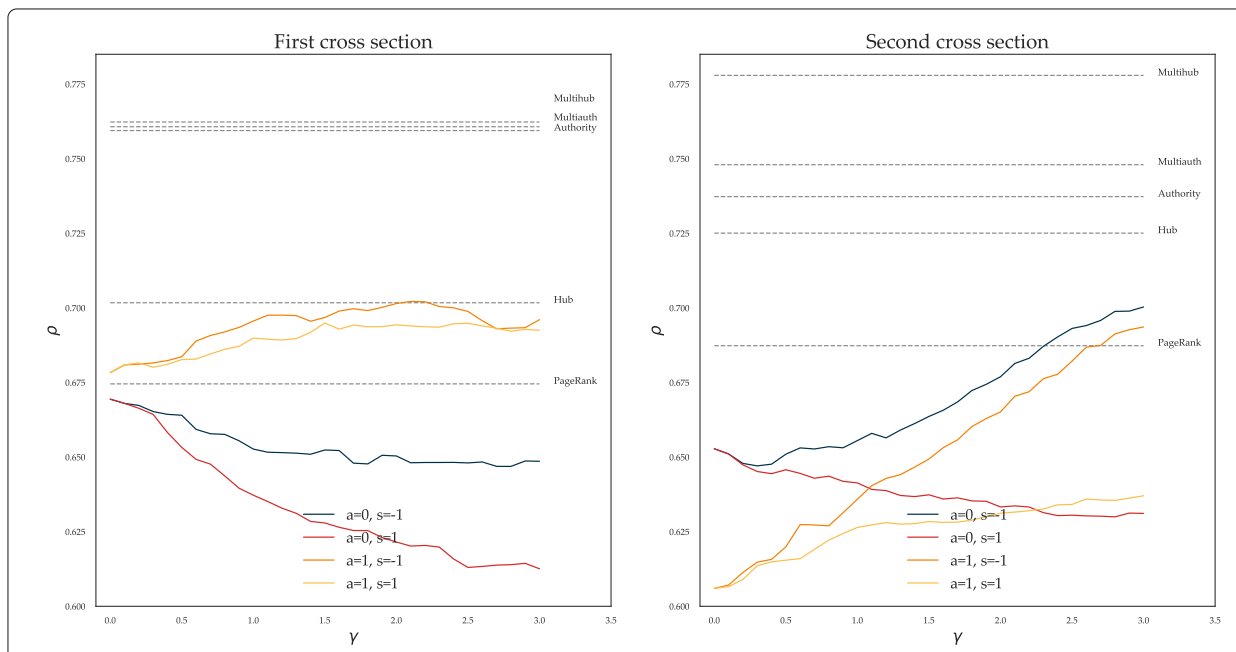

Fig. 19 Spearman correlation of rankings of different measures of node centrality with respect to ranking by pcGDP 


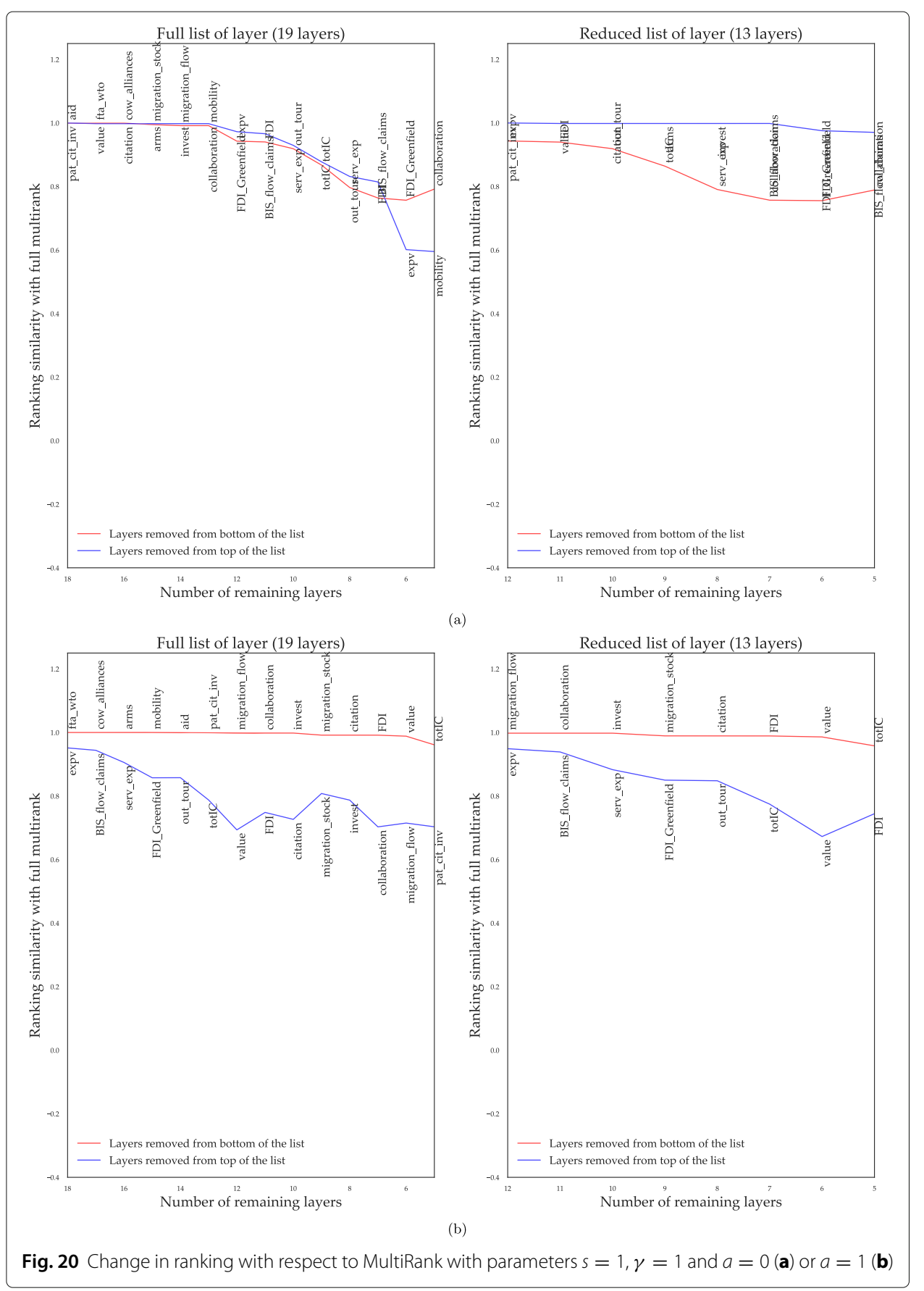

data. However we do not aim to completely reconstruct the ranking of nodes by pcGDP, otherwise our measures will not have an informative content.

Our test for these last two criteria is shown in Fig. 19. Here we have plotted the Spearman rank correlation coefficient $(\rho)$ between the nodes ranking resulting from pcGDP and the one resulting from different centrality algorithms while we let the parameter $\gamma$ take different values ( $\mathrm{x}$-axis). The coloured lines are 4 different configuration of the MultiRank while the dashed lines are the MD-HITS hubs and authority scores (here called MultiHub and MultiAuth) which by definition are not affected by $\gamma$. For robustness we 


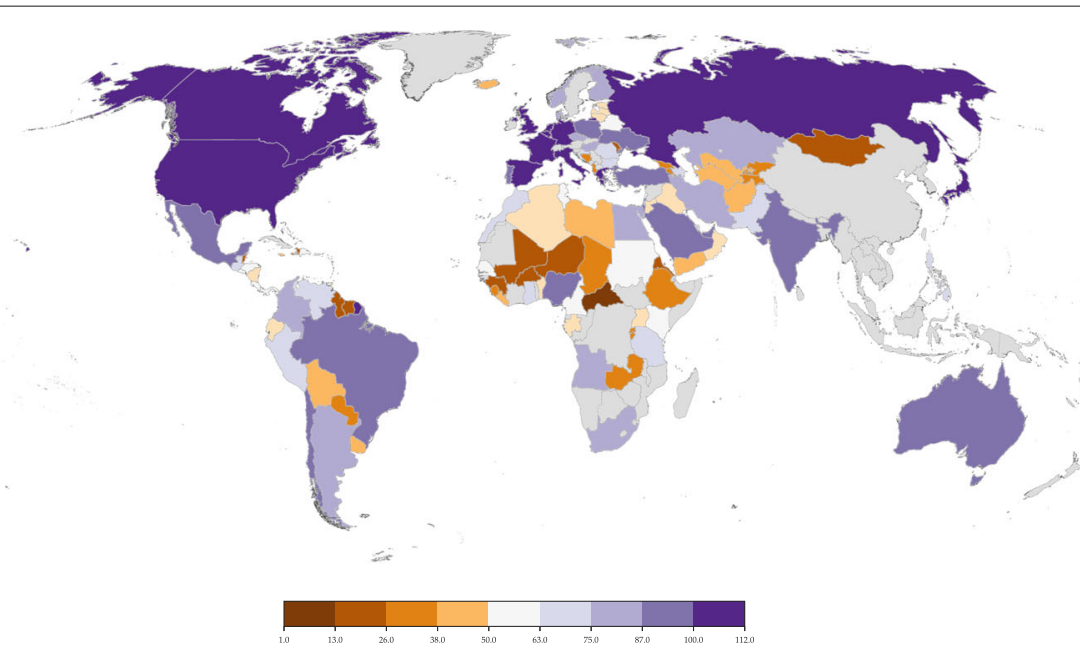

(a)

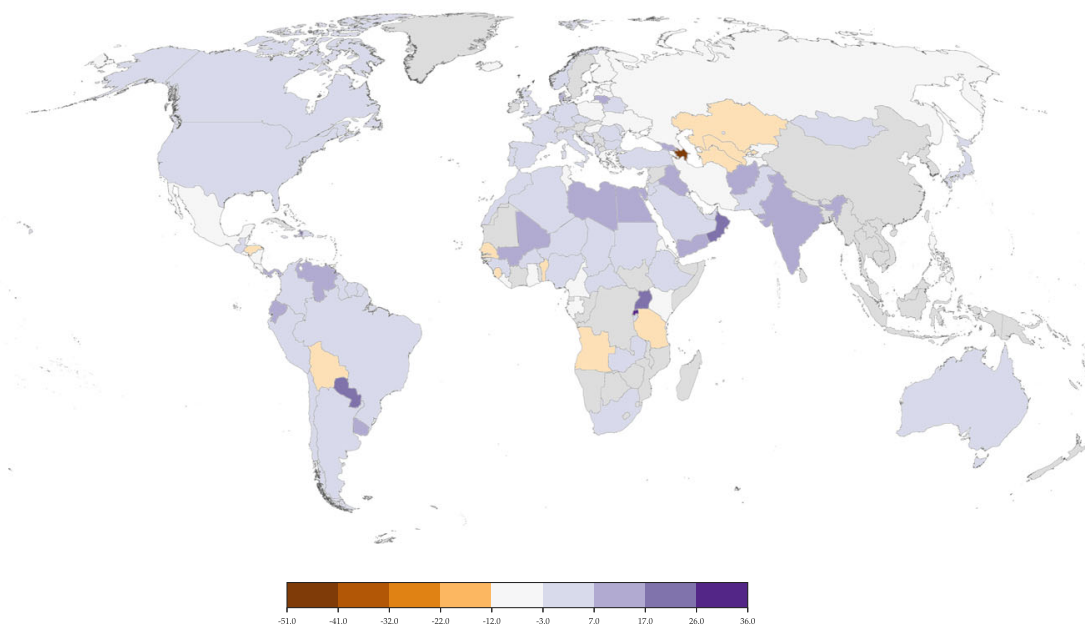

(b)

Fig. 21 Geographic distribution of the multiplex hub score (MultiHub). Cross section in 2003 (a) and change of the ranking from 2003 to 2010 (b)

have added the single layer version of the three previous measures (PageRank, Hubs and Authority) calculated on the aggregate multiplex obtained by summing over all the layers the corresponding entries of their adjacency matrices, which are constant too.

The first thing to notice is that the ranking deriving from hub scores for the MD-HITS algorithm is the one which follows more closely the pcGDP ranking with a Spearman $\rho$ between 0.76 and 0.78 . In the cross section in 2003 also the MD-HITS authority scores and the authority scores calculated on the aggregated network have a good fit too but the same strong similarity does not hold in the cross section in 2010.

Moreover among the ranking obtained from the MultiRank we see different behaviours according to different choice of parameters in the two cross sections: only for some particular combinations of them in the 2003 cross section the MultiRank outperforms the ranking resulting from PageRank calculated on the aggregated multiplex while in the 2010 cross section for the majority of the values of $\gamma$ the ranking resulting from PageRank is better than all possible combination of the MultiRank making it the worse choice. 


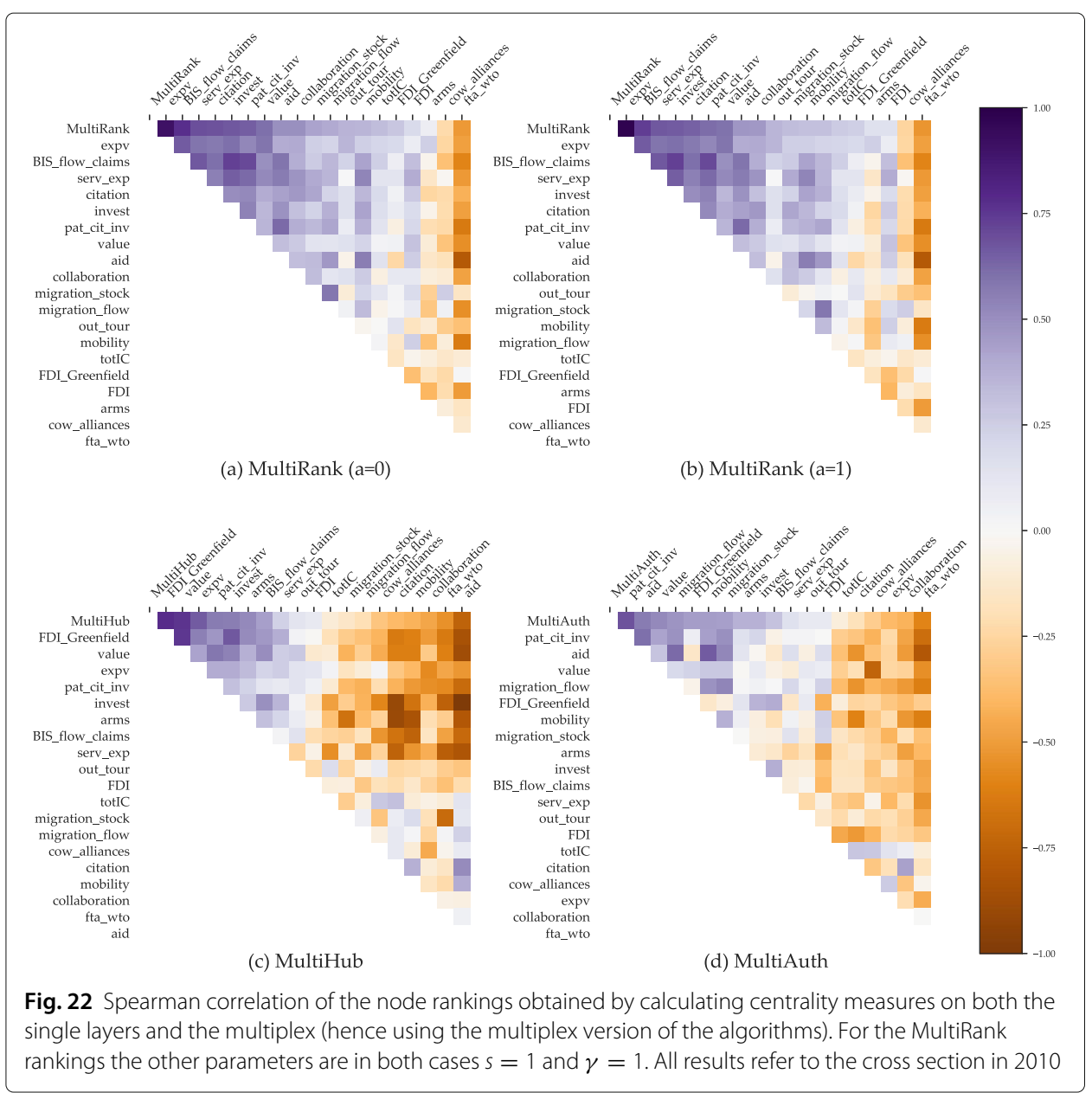

We observe in fact that in the 2003 cross section the combinations with $a=1$ are rewarded, while for $a=0$ the Spearman correlation with pcGDP ranking is lower. On the contrary in the second cross section combination with $s=-1$ are rewarded irrespective of the magnitude of $a$.

Even though in these last cases we obtain a better fit with respect to pcGDP, still choosing $s=-1$ which corresponds to assume that low centrality nodes are more important is difficult to justify without further evidence and does not account for a sufficient improvement in fitting in cross section 2010. Moreover the combination $a=1, s=-1$ which would be ideal in both cross section is the more affected by the choice of $\gamma$ in cross section 2010, hence making it too volatile. Finally for $\gamma=1$ has the same fitting to pcGDP ranking as our second choice $a=0, s=1$.

Hence we have two configurations of the MultiRank which are worth analyzing since they stem from sensible assumptions on the data and are sufficiently stable with respect to $\gamma$. Once we fix the parameter $\gamma$ to 1 , one of them, $(a=1, s=1)$, has the best fitting with respect to pcGDP in cross section 2003, while the other $(a=0, s=1)$ represents a good second best option in cross section 2010. Similar trade-offs could have arises by choosing one of the other combinations, which however have stronger implication that needs further empirical justifications. 


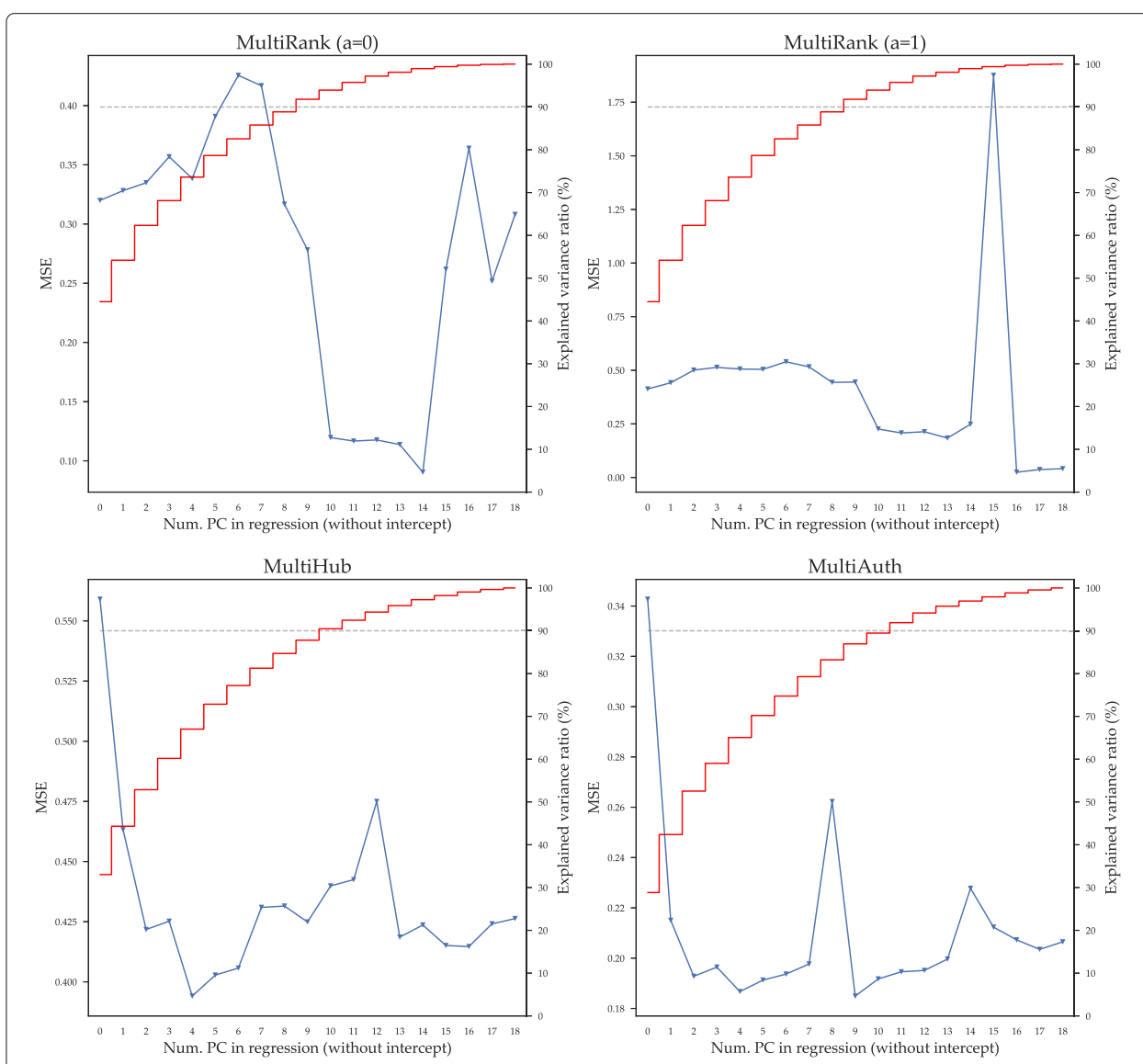

Fig. 23 In red: number of principal components sufficient to explain $90 \%$ of the variance of the measures of centrality calculated on the single layers (scale reported on the right $y$-axis). In blue: mean square error obtained by regressing the multilayer measures of centrality against the principal components of the single layer centralities, added one after one (scale reported on the left $y$-axis). All results refer to the cross section in 2010 


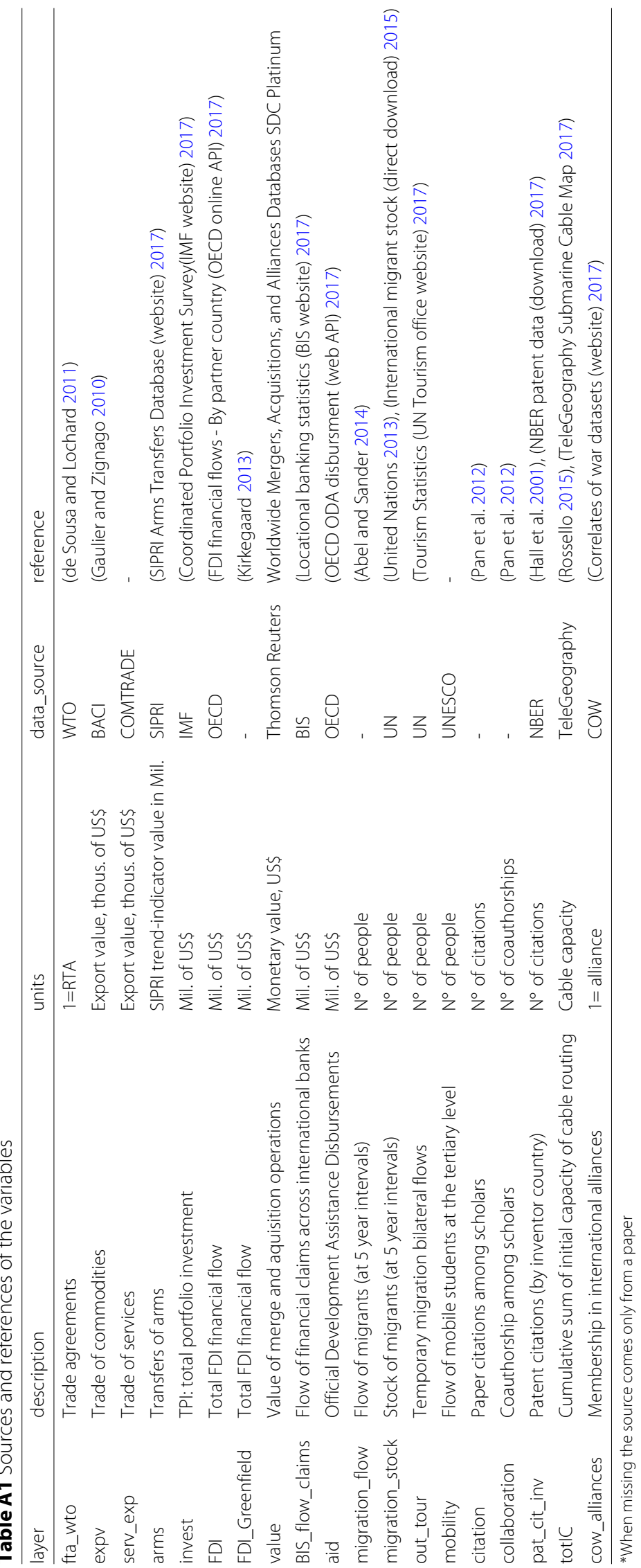




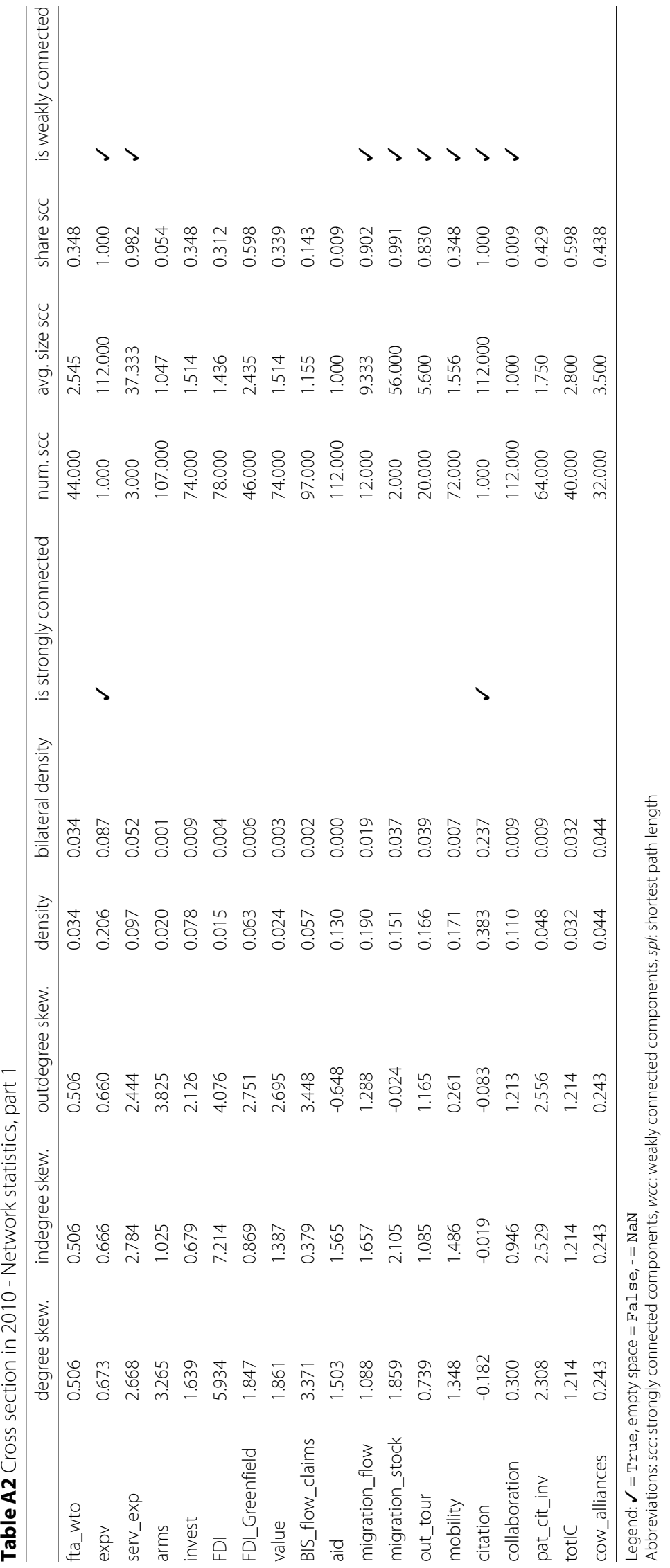




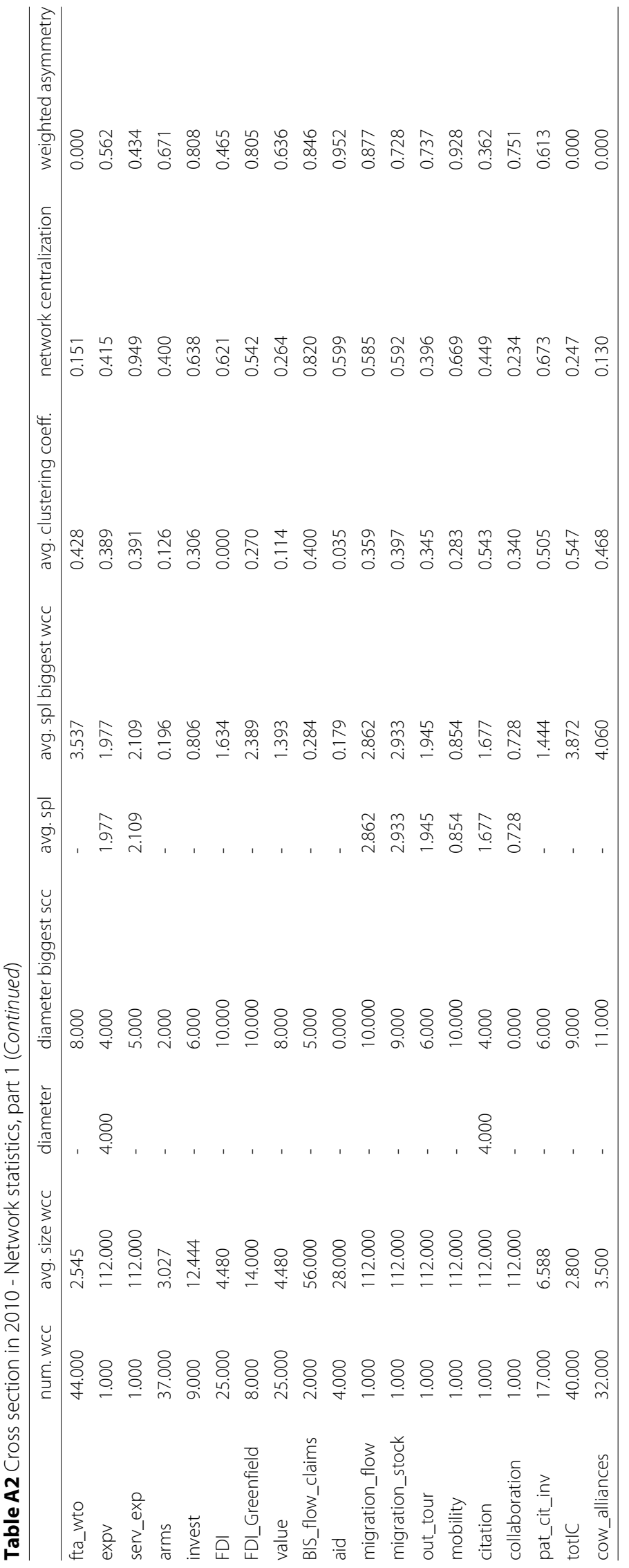


Authors' information (optional)

Not applicable.

\section{Abbreviations}

GDP: gross domestic product; PPP: purchasing power parity; HITS: hyperlink-induced topic search; MD-HITS: multidimensional HITS; pcGDP: per capita GDP; M\&A:merger and acquisition; FDI: foreign direct investment; COW: correlates of war; TPI: total portfolio investment; ODA: official development assistance; BIS: bank of international settlements; scc: strongly connected component; wcc: weakly connected component; spl: shortest path length

\section{Acknowledgements}

Not applicable

\section{Authors' contributions}

GB performed the analysis. GB, MR and GF wrote the manuscript. All authors reviewed and approved the manuscript.

\section{Funding}

Not applicable.

\section{Availability of data and materials}

The datasets analyzed during the current study are publicly available. Detailed sources and link are reported in Table A2. A version of the dataset is available also at: https://github.com/gibbbone/international_multiplex_network

\section{Ethics approval and consent to participate}

Not applicable.

\section{Consent for publication}

Not applicable.

\section{Competing interests}

The authors declare that they have no competing interests.

\section{Author details}

${ }^{1}$ IMT School of Advanced Studies, Lucca, 55100, Lucca, Italy. ${ }^{2}$ Sant'Anna School of Advanced Studies, Pisa, 56122, Pisa, Italy. ${ }^{3}$ Centre d'Etudes Prospectives et d'Informations Internationales, Paris, 75334, Paris, France.

Received: 29 March 2019 Accepted: 6 September 2019

Published online: 26 December 2019

\section{References}

Abel G (2013) Estimating global migration flow tables using place of birth data. Demogr Res 28:505-546

Abel GJ, Sander N (2014) Quantifying Global International Migration Flows. Science 343(6178):1520-1522. https://doi. org/10.1126/science.1248676

Aleta A, Moreno Y (2019) Multilayer Networks in a Nutshell. Ann Rev Condens Matter Phys 10(1):45-62. https://doi.org/10 1146/annurev-conmatphys-031218-013259

Armenter R, Koren M (2014) A Balls-and-Bins Model of Trade. Am Econ Rev 104(7):2127-2151. https://doi.org/10.1257/aer 104.7.2127. http://pubs.aeaweb.org/doi/10.1257/aer.104.7.2127

Arrigo F, Tudisco F (2018) Multi-Dimensional, Multilayer, Nonlinear and Dynamic HITS. arXiv:180908004 [physics]. http:// arxiv.org/abs/1809.08004

Bardoscia M, Battiston S, Caccioli F, Caldarelli G (2015) DebtRank: A Microscopic Foundation for Shock Propagation. PLOS ONE 10(6):e0130,406. https://dx.plos.org/10.1371/journal.pone.0130406

Battiston F, Nicosia V, Latora V (2014) Structural measures for multiplex networks. Phys Rev E 89(3). https://link.aps.org/ doi/10.1103/PhysRevE.89.032804

Battiston S, Puliga M, Kaushik R, Tasca P, Caldarelli G (2012) DebtRank: Too Central to Fail? Financial Networks, the FED and Systemic Risk. Scientific Reports 2(1). http://www.nature.com/articles/srep00541

Bayer R (2006) Diplomatic Exchange Data Set, v2006.1 edn. Correlates of War Project. http://correlatesofwar.org/

Boccaletti S, Bianconi G, Criado R, del Genio C, Gómez-Gardeñes J, Romance M, Sendiña-Nadal I, Wang Z, Zanin M (2014) The structure and dynamics of multilayer networks. Phys Rep 544(1):1-122. http://linkinghub.elsevier.com/retrieve/ pii/S0370157314002105

Bródka P, Chmiel A, Magnani M, Ragozini G (2018) Quantifying layer similarity in multiplex networks: a systematic study. R Soc Open Sci 5(8):171,747. https://royalsocietypublishing.org/doi/full/10.1098/rsos.171747

Brin S, Page L (1998) The anatomy of a large-scale hypertextual Web search engine. Comput Netw ISDN Syst 30(1):107-117. http://www.sciencedirect.com/science/article/pii/S016975529800110X

Campi M, Dueeas M, Barigozzi M, Fagiolo G (2016) Do Intellectual Property Rights Influence Cross-Border Mergers and Acquisitions?. SSRN Electron J. https://www.ssrn.com/abstract=2805067

Chaney T (2014) The Network Structure of International Trade. Am Econ Rev 104(11):3600-3634. http://pubs.aeaweb.org/ doi/10.1257/aer.104.11.3600

Colizza V, Barrat A, Barthélemy M, Vespignani A (2006) The role of the airline transportation network in the prediction and predictability of global epidemics. Proc Natl Acad Sci U S A 103(7):2015-2020

Coordinated Portfolio Investment Survey(IMF website) (2017) Coordinated Portfolio Investment Survey. http://data.imf. org/regular.aspx?key=60587804, published: Website 
Correlates of war datasets (website) (2017) Correlates of war datasets. http://www.correlatesofwar.org/data-sets, published: Website

Dasgupta P, Mäler KG (2000) Net national product, wealth, and social well-being. Environ Dev Econ 5(1):69-93. http:// www.journals.cambridge.org/abstract_S1355770X00000061

De Bacco C, Power EA, Larremore DB, Moore C (2017) Community detection, link prediction, and layer interdependence in multilayer networks. Phys Rev E 95(4). http://link.aps.org/doi/10.1103/PhysRevE.95.042317

De Domenico M, Nicosia V, Arenas A, Latora V (2015a) Structural reducibility of multilayer networks. Nat Commun 6(1). http://www.nature.com/articles/ncomms7864

De Domenico M, Solé-Ribalta A, Omodei E, Gómez S, Arenas A (2015b) Ranking in interconnected multilayer networks reveals versatile nodes. Nat Commun 6(1). http://www.nature.com/articles/ncomms7868

Easley D, Kleinberg J (2010) Networks, Crowds, and Markets: Reasoning about a Highly Connected World. 1st edn. Cambridge University Press, New York

Fagiolo G, Mastrorillo M (2014) Does Human Migration Affect International Trade? A Complex-Network Perspective. PLoS ONE 9(5):e97,331. http://dx.plos.org/10.1371/journal.pone.0097331

Fagiolo G, Santoni G (2015) Human-mobility networks, country income, and labor productivity. Netw Sci 3(03):377-407. http://www.journals.cambridge.org/abstract_S2050124215000259

Fagiolo G, Santoni G (2016) Revisiting the role of migrant social networks as determinants of international migration flows. Appl Econ Lett 23(3):188-193

FDI financial flows - By partner country (OECD online API) (2017) FDI financial flows. http://stats.oecd.org/Index.aspx? Queryld=64194, published: Website

Gaulier G, Zignago S (2010) BACI: International Trade Database at the Product-Level. The 1994-2007 Version. Tech Rep 2010-23. CEPII research center

Gemmetto V, Cardillo A, Garlaschelli D (2017) Irreducible network backbones: Unbiased graph filtering via maximum entropy. arXiv:170600230 [physics]

Gibler DM (2008) International Military Alliances, 1648-2008. CQ Press

Hafner-Burton EM, Kahler M, Montgomery AH (2009) Network Analysis for International Relations. Int Organ 63(3):559-592. https://www.cambridge.org/core/journals/international-organization/article/network-analysis-forinternational-relations/DE2910979C1B5C44C4CC13F336C5DE97

Hall BH, Jaffe AB, Trajtenberg M (2001) The NBER Patent Citation Data File: Lessons, Insights and Methodological Tools Working Paper 8498, National Bureau of Economic Research. https://doi.org/10.3386/w8498

Halu A, Mondragón RJ, Panzarasa P, Bianconi G (2013) Multiplex PageRank. PLoS ONE 8(10):e78,293. http://dx.plos.org/10. 1371/journal.pone.0078293

lacovacci J, Bianconi G (2016) Extracting Information from Multiplex Networks. Chaos: An Interdisciplinary Journal of Nonlinear Science 26(6):065,306. http://arxiv.org/abs/1602.08751

lacovacci J, Rahmede C, Arenas A, Bianconi G (2016) Functional Multiplex PageRank. EPL (Europhysics Lett) 116(2):28,004. http://stacks.iop.org/0295-5075/116/i=2/a=28004?key=crossref.4c0ed62a515e4c93404e6e7de9476d6d

International migrant stock (direct download) (2015) International migrant stock (direct download). http://www.un.org/ en/development/desa/population/migration/data, published: Website

Kali R, Horowitz A, Song H (2017) Rethinking the Aid-Growth Relationship: A Network Approach

Kaluza P, Kölzsch A, Gastner MT, Blasius B (2010) The complex network of global cargo ship movements. J R Soc Interface 7(48):1093-1103

Kirkegaard J (2013) New Avenues for Empirical Analysis of Cross-Border Investments: An Application for the ASEAN Members and Middle and Low Income Country Outward Investments. PhD Thesis. Johns Hopkins University, Baltimore

Kivela M, Arenas A, Barthelemy M, Gleeson JP, Moreno Y, Porter MA (2014) Multilayer networks. J Compl Netw 2(3):203-271. https://academic.oup.com/comnet/article-lookup/doi/10.1093/comnet/cnu016

Kleinberg JM (1999) Hubs, authorities, and communities. ACM Comput Surv 31(4es):5-es. http://portal.acm.org/citation. $\mathrm{cfm}$ ?doid $=345966.345982$

Lange GM, Wodon Q (2018) The Changing Wealth of Nations 2018: Building a Sustainable Future. The World Bank. http:// elibrary.worldbank.org/doi/book/10.1596/978-1-4648-1046-6

Lee KM, Kim JY, Cho Wk, Goh KI, Kim IM (2012) Correlated multiplexity and connectivity of multiplex random networks. New J Phys 14(3):033,027. http://stacks.iop.org/1367-2630/14/i=3/a=033027?key=crossref. 7734afd83ce1f03a4b0fdcb967388e36

Locational banking statistics (BIS website) (2017) Locational banking statistics. http://www.bis.org/statistics/bankstats. htm? $\mathrm{m}=6 \% 7 \mathrm{C} 31 \% 7 \mathrm{C} 69$, published: Website

Metulini R, Sgrignoli P, Schiavo S, Riccaboni M (2018) The network of migrants and international trade. Econ Politica 35(3):763-787

Minoiu C, Reyes JA (2013) A network analysis of global banking: 1978-2010. J Financ Stab 9(2):168-184. http://linkinghub. elsevier.com/retrieve/pii/S1572308913000193

NBER patent data (download) (2017) NBER patent data. https://sites.google.com/site/patentdataproject/Home/ downloads, published: Website

OECD ODA disbursment (web API) (2017) OECD ODA disbursment. http://stats.oecd.org/Index.aspx?DataSetCode= Table2A, published: Website

Pan RK, Kaski K, Fortunato S (2012) World citation and collaboration networks: Uncovering the role of geography in science. Sci Rep 2:902

Pevehouse J, Nordstrom T, Warnke K (2003) Intergovernmental Organizations, 1815-2000: A New Correlates of War Data Set. 2nd edn. Correlates of War Project. http://correlatesofwar.org

Radicchi F, Ramasco JJ, Fortunato S (2011) Information filtering in complex weighted networks. Phys Rev E Stat Nonlinear Soft Matter Phys 83(4 Pt 2):046,101

Rahmede C, lacovacci J, Arenas A, Bianconi G (2018) Centralities of nodes and influences of layers in large multiplex networks. J Complex Netw 6(5):733-752. https://academic.oup.com/comnet/article/6/5/733/4561631

Riccaboni M, Rossi A, Schiavo S (2013) Global networks of trade and bits. J Econ Interac Coord 8(1):33-56. http://link. springer.com/10.1007/s11403-012-0101-x 
Rossello G (2015) Internet Infrastructure: a Multi-layer Network Analysis, Master's thesis. University of Pisa Sen A (1985) Commodities and Capabilities. North-Holland, Amsterdam

Serrano MÁ, Boguñá M, Vespignani A (2009) Extracting the multiscale backbone of complex weighted networks. Proc Natl Acad Sci U S A 106(16):6483-6488

Sgrignoli P, Metulini R, Schiavo S, Riccaboni M (2015) The relation between global migration and trade networks. Physica A: Statistical Mechanics and its Applications 417:245-260. http://inkinghub.elsevier.com/retrieve/pii/ S037843711400795X

SIPRI Arms Transfers Database (website) (2017) SIPRI Arms Transfers Database. https://www.sipri.org/databases/ armstransfers, published: Website

Smith DA, White DR (1992) Structure and Dynamics of the Global Economy: Network Analysis of International Trade 1965-1980. Soc Forces 70(4):857. http://www.jstor.org/stable/2580193?origin=crossref

de Sousa J, Lochard J (2011) Does the Single Currency Affect Foreign Direct Investment?*. Scand J Econ 113(3):553-578

Stevenson B, Wolfers J (2013) Subjective Well-Being and Income: Is There Any Evidence of Satiation?. Am Econ Rev 103(3):598-604. http://pubs.aeaweb.org/doi/10.1257/aer.103.3.598

TeleGeography Submarine Cable Map (2017) TeleGeography Submarine Cable Map. https://www.submarinecablemap. com/, published: Website

Tourism Statistics (UN Tourism office website) (2017) Tourism Statistics. http://www.e-unwto.org/loi/unwtotfb, published: Website

United Nations PopulationDivision (2013) United Nations PD (2013) Trends in International Migrant Stock: Migrants by Destination and Origin, The 2013 Revision

\section{Publisher's Note}

Springer Nature remains neutral with regard to jurisdictional claims in published maps and institutional affiliations.

Submit your manuscript to a SpringerOpen ${ }^{\circ}$ journal and benefit from:

- Convenient online submission

Rigorous peer review

- Open access: articles freely available online

- High visibility within the field

- Retaining the copyright to your article

Submit your next manuscript at $\boldsymbol{\triangleright}$ springeropen.com 